\title{
Réflectivité hors spéculaire et diffraction de surface
}

\author{
F. Ott
}

Laboratoire Léon Brillouin, CEA/CNRS, bâtiment 563, CEA/Saclay, 91191 Gif-sur-Yvette,
France

\begin{abstract}
Résumé : La technique de réflectivité de neutrons permet d'obtenir des informations structurelles et magnétiques sur des systèmes multicouches. Dans la géométrie standard de réflectivité spéculaire il est possible d'obtenir des informations sur le profil de densité de longueur de diffusion ou le profil d'aimantation suivant la direction perpendiculaire au plan du système multicouche. Dans ce cours nous allons présenter les différents aspects de la diffusion hors spéculaire en tefflectivité de neutrons et montrer de quelle manière elle permet d'accéder à des informations planaires à la fois morphologiques et magnétiques. Nous présenterons les différentes origines de la diffusion hors spéculaire (dans les cas magnétiques et non magnétiques), puis nous décrirons les formalismes permettant de décrire ces phénomènes et enfin nous discuterons quelques exemples de mesures expérimentales sur des systèmes magnétiques.
\end{abstract}

\section{INTRODUCTION}

Dans ce chapitre, nous allons décrire l'utilisation de la réflectivité hors spéculaire pour l'étude de systèmes de couches minces. Comme cela a été vu au chapitre précédent, la réflectivité de neutrons spéculaire permet d'obtenir des informations sur les profils d'aimantation dans des systèmes multicouches. Dans ces mesures de réflectivité, on s'intéresse uniquement à la diffusion dans la direction spéculaire c'est à dire dans la direction de réflexion telle que l'angle d'incidence du faisceau de neutrons sur l'échantillon est égal à l'angle de réflexion. Dans cette géométrie, on rappelle que :

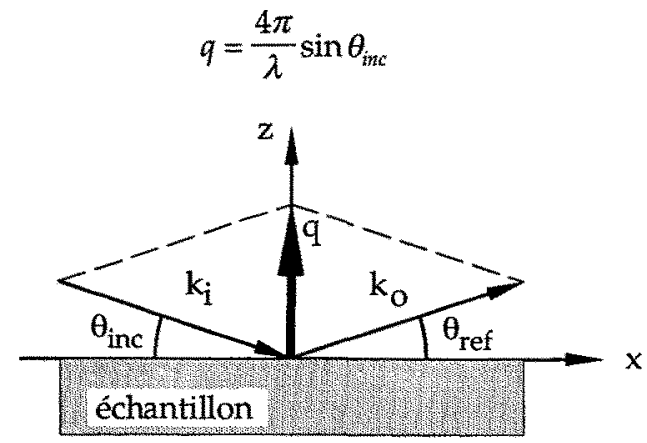

Figure 1 : gémétrie de réflexion spéculaire: $\boldsymbol{k}_{\xi}$ est le vecteur d'onde du faisceau incident, $\boldsymbol{k}_{0}$ est le vecteur d'onde de l'onde réfléchie, $\boldsymbol{q}=\boldsymbol{k}_{r a f}-\boldsymbol{k}_{\text {ine }}$ est le vecteur de diffusion. Dans le cas d'une réflexion spéculaire, $q$ est dirigé suivant $(\mathrm{Oz})$. 
Les mesures de réflectivité supposent qu'il y invariance du système étudié par translation dans le plan (xOy) c'est à dire que le potentiel d'interaction $\mathbf{V}$ est uniquement fonction de $z$. Dans ce cas et uniquement dans ce cas, la diffusion sur l'échantillon sera parfaitement spéculaire Cependant, si le potentiel d'interaction $\mathbf{V}$ est une fonction de $x$ ou $y$, cela donne naissance à de la diffusion dans les directions hors de la direction spéculaire. La mesure de cette diffusion hors spéculaire pourra donner des informations sur la structure du système étudié dans le plan (xOy).

\section{D'OU PROVIENT LA DIFFUSION HORS SPECULAIRE?}

\subsection{Imperfection des systèmes de couches minces}

Les systèmes étudiés par réflectivité spéculaire de neutrons (voir cours précédent) ne sont jamais parfaits. Les surfaces ne présentent jamais une invariance de translation idéale. Les couches présentent une certaine rugosité qui les écarte plus ou moins du modèle idéal. Les raisons sont variées: interdiffusion entre les couches du système multicouche, rugosité intrinsèque lors de la croissance de la couche, ondes capillaires de surface...

Cela donne lieu à de la diffusion hors spéculaire. Cette diffusion hors spéculaire doit être prise en compte afin de pouvoir interpréter quantitativement les mesures expérimentales de réflectivité.

On peut considérer qu'il existe trois échelles de rugosité caractérisée par une longueur caractéristique latérale $\xi$ :

- une rugosité au niveau atomique. Elle est typiquement liée à l'interdiffusion entre les couches

minces,

$\xi<100 \mathrm{~nm}$.

- une rugosité intermédiaire ( $\xi$ de $0.5 \mu \mathrm{m}$ à $50 \mu \mathrm{m}$ ). Elle donne lieu à une diffusion non spéculaire. Un traitement correct des courbes de réflectivité exige que cette diffusion non spéculaire soit mesurée afin de corriger le signal de réflectivité spéculaire

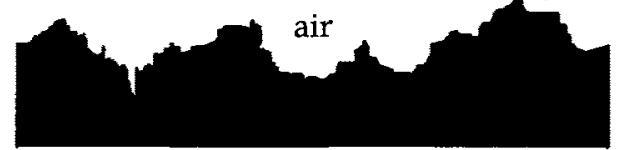

- une rugosité à grande échelle $(\xi>50 \mu \mathrm{m})$. Elle correspond par exemple à des variations d'épaisseur le long de l'échantillon (cela peut être particulièrement important dans le cas d'échantillons de grande surface).

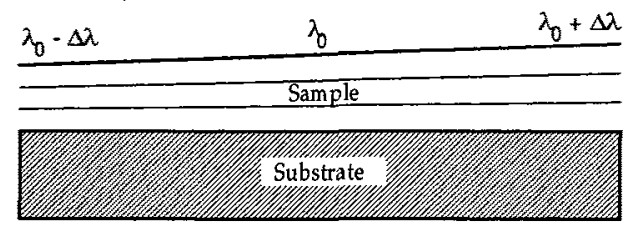

Ces trois types de "rugosité » ont cependant des effets très différents sur le signal de réflectivité spéculaire (voir cours précédent). On peut décrire de manière satisfaisante l'interdiffusion atomique en introduisant au niveau de l'interface une couche d'alliage qui possède un indice moyen entre les 
couches supérieure et inférieure. L'ajustement numérique permet d'évaluer la composante de cet alliage d'interface en pourcentages des deux espèces chimiques.

Dans une mesure de réflectivité, on se place dans l'approximation de milieu continu (voir démonstration Bergevin [1]). Dans une première approximation, cette rugosité d'interdiffusion ne donne pas naissance à un signal hors spéculaire car le potentiel d'interaction $\mathbf{V}$ est moyenné dans le plan (xOy). S'il y a interdiffusion à l'interface, on passera typiquement d'un potentiel $\boldsymbol{V}(z)$ ayant une variation abrupte à un potentiel moyen $\langle\boldsymbol{V}(z)\rangle$ présentant une variation plus douce au niveau de l'interface.

\subsection{Etudes de petites structures à l'échelle du nanomètre ou du micromètre}

Dans le paragraphe précédent, nous avons considérế la diffusion hors spéculaire due à la rugosité comme une complication supplémentaire dans l'interprétation des courbes de réflectivité spéculaire. On peut au contraire adopter l'approche inverse et considérer que cette diffusion hors spéculaire peut donner des informations sur la structure du matériau ou de la couche étudiée dans le plan de la couche mince. L'êtude de cette diffusion hors spéculaire peut être vue comme l'extension des études de diffusion aux petits angles (SANS) à des systèmes en couches minces. Le principe de mesure reste cependant le même. II faut cependant noter que les propriétés des systèmes sous la forme d'un échantillon « massif » changent en général lorsque celui ci est déposé sous la forme de couche mince.

On montrera dans la suite que les tailles des objets ou des structures que l'on peut étudier dans une géométrie de réflectivité s'étagent de quelques nanomètres à quelques dizaines de nanomètres.

\subsubsection{Structures non magnétiques}

Comme il a été dit précédemment, toutes les études de diffusion aux petits angles peuvent être étendues au cas des couches minces. Les problèmes dans les systèmes de couches minces sont cependant souvent spécifiques. Quelques exemples d'etudes sont : mesure de la corrosion de films minces métalliques. (piquage des surfaces par ex.), structures bidimensionnelles dans des multicouches de polymères (auto-organisation en îlots par ex.)..

\subsubsection{Structures magnétiques}

L'utilisation de neutrons polarisés permet d'étudier des nanostructures magnétiques. On peut citer par exemple : réseau de particules magnétiques auto-organisées en réseau $2 \mathrm{D}$ (particules de cobalt ou de ferrite), réseau de domaines magnétiques, structures en domaines magnétiques, petites structures magnétiques fabriquées artificiellement (par lithographie)... 

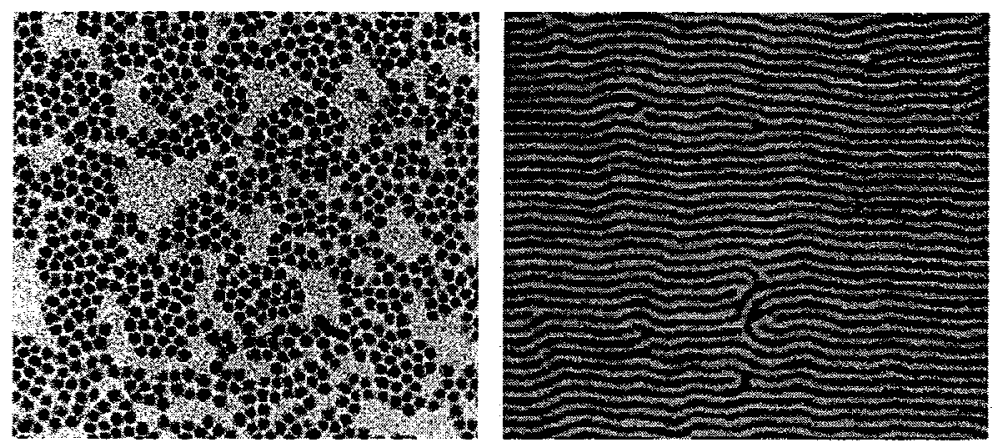

Figure 2: A gauche, réseau bidimensionnel de petites particules de cobalt; A droite réseau de domaines magnétiques dans une couche de $F e_{0 .} P d_{0.5}$.

\subsubsection{Extension à l'étude de structures à l'échelle atomique: diffraction de surface}

En géométrie de réflectivité (incidence rasante) il est possible de mesurer la diffusion à grand angle. Il est ainsi possible de sonder l'ordre magnétique à l'échelle atomique dans des films minces (qqes $10 \mathrm{~nm}$ d'épaisseur).

\section{GÉOMÉTRIES DE DIFFUSION HORS SPÉCULAIRE ET DE DIFFRACTION DE SURFACE}

\subsection{Diffusion hors spéculaire en géométrie de réflectivité (petits angles)}

Dans ce paragraphe, nous allons décrire la géométrie typique de diffusion hors spéculaire et fixer les notations. Considérons un échantillon placé verticalement (voir

Figure 3). Cet échantillon définit le plan (xOy). La direction $(\mathrm{Oz})$ est la direction perpendiculaire à l'échantillon. $(\mathrm{Ox})$ est la direction de propagation des neutrons. Soit $\theta_{i}$ l'angle d'incidence, soit $\theta_{o}$ l'angle de réflexion spécualire, soit $\Delta \theta_{x}$ l'angle entre la direction de diffusion et la direction de réflexion spéculaire mesurée dans le plan d'incidence, soit $\Delta \theta_{y}$ l'angle entre la direction de diffusion et la direction de réflexion spéculaire mesurée dans le plan perpendiculaire au plan d'incidence.

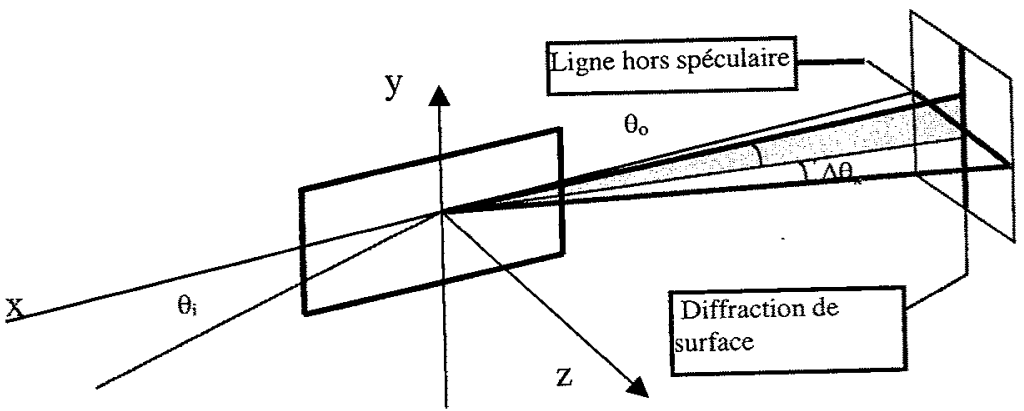

Figure 3 : géométrie de diffusion hors spéculaire et géométrie de diffraction de surface. 
Les vecteurs d'onde incident et diffusé sont définis (au premier ordre en $k_{0}$ ) par;

$$
\mathbf{k}_{i}\left|\begin{array}{c}
k_{0} \cos \theta_{i} \\
0 \\
-k_{\theta} \sin \theta_{i}
\end{array}\right| \text { and } \quad \mathbf{k}_{o}\left|\begin{array}{c}
k_{0} \cos \theta_{\theta} \cos \Delta \theta_{y} \\
k_{0} \sin \Delta \theta_{y} \\
k_{0} \sin \theta_{o}
\end{array}\right|
$$

où $\mathrm{k}_{0}=2 \pi / \lambda$ est la norme du vecteur d'onde du neutron

Le vecteur de diffusion $q$ défini par $q=k_{0}-k_{j}$ est donné par:

(on suppose que tous les angles sont petits ; on rappelle que $\theta_{i}=\theta_{0}$ ):

$$
\left|\begin{array}{c}
k_{0} \theta_{i} \Delta \theta_{x} \\
k_{0} \Delta \theta_{y} \\
2 k_{0} \theta_{i}
\end{array}\right|
$$

Les tailles latérales qui peuvent être sondếes sont typiquement données par $2 \pi / q_{x}$ et $2 \pi / q_{y}$. Ces deux tailles sont d'ordre de grandeur très différents. La raison essentielle est due au fait que l'on travaille en incidence rasante que cela introduit le facteur $\theta_{i}$ dans l'expression de $q_{x}$. L'angle $\theta_{i}$ est typiquement de l'ordre de $10^{2}$ rad.

\subsection{Application numérique}

Sur le réflectomètre PRISM:

- longueur d'onde monochromatique de $0.4 \mathrm{~nm}$

- meilleure résolution angulaire $0.02^{\circ}$

- angle max.: $10^{\circ}$ (en pratique, pas assez de flux au-dessus)

Vérifier que: $10 \mathrm{~mm}<2 \pi / q_{y}<400 \mathrm{~nm}$ and $1 \mathrm{\mu m}<2 \pi / q_{x}<50 \mathrm{hm}$.

La limite supérieure est donnée par la résolution angulaire du réflectomètre et la limite inférieure par la limite en intensité disponible. On constate donc que l'on pourra sonder des tailles d'objets très différentes en fonction que l'on mesure l'intensité hors spéculaire dans le plan d'incidence ou dans le plan perpendicularre au plan d'incidence.

\section{1 .2 Diffusion hors spéculaire dans le plan d'incidence}

En diffusion hors spéculaire, le vecteur de diffusion $q$ n'est plus aligne le long de l'axe (Ox), une composante $\mathrm{q}_{\mathrm{x}}$ apparait. La Figure 4 définit la géométrie de diffusion hors spéculaire dans le plan d'incidence. 


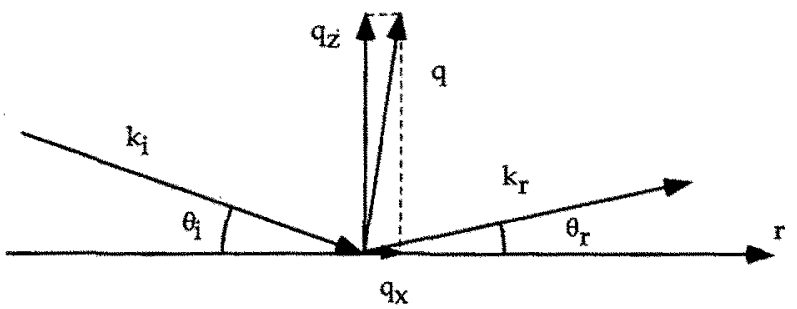

Figure 4: géométrie de diffusion non spéculaire. Le vecteur de diffusion $q$ possède une composante $q_{x}$

Les composantes du vecteur de diffusion $\mathbf{q}$ sont données par des expressions symétriques:

$$
\begin{aligned}
& q_{z}=\frac{4 \pi}{\lambda} \sin \left(\frac{\theta_{i}+\theta_{r}}{2}\right) \cos \left(\frac{\theta_{i}-\theta_{r}}{2}\right) \\
& q_{x}=\frac{4 \pi}{\lambda} \sin \left(\frac{\theta_{i}+\theta_{r}}{2}\right) \sin \left(\frac{\theta_{i}-\theta_{r}}{2}\right)
\end{aligned}
$$

Ces expressions se révèlent particulièrement utiles lorsque l'on souhaite passer de l'espace réel $\left(\theta_{i}, \theta_{o}\right)$ à l'espace réciproque $\left(q_{x}, q_{z}\right)$ et inversement.

\subsubsection{Diffusion hors spéculaire dans le plan perpendiculaire au plan d'incidence}

Dans cette géométrie on se retrouve dans une situation tout à fait similaire à celle d'une expérience de diffusion aux petits angles (voir cours sur le SANS) à la seule différence que l'on s'intéresse à des systèmes en couches minces. Cependant on montrera que dans une géométrie de réflectivité (réflexion en incidence rasante), la sensibilité aux structures dans le plan du film est beaucoup plus importante que si l'on effectuait une mesure avec le faisceau de neutrons perpendiculaire à l'échantillon par exemple.

\subsection{Géométrie de diffraction de surface à grand angle}

Comme cela a été évoqué précédemment, il est possible de mesurer la diffraction à grand angle sur une couche mince. Il est nécessaire de disposer d'un multidétecteur. Ce type de spectromètre est relativement peu courant car il exige une collimation dans les deux directions de l'espace. Le plan d'incidence est défini par $\left(k_{i}, O z\right)$. Si la structure de l'échantillon est monocristalline, il est possible d'observer un signal de diffraction de surface dans la direction $\mathbf{k}_{\mathbf{f}}$. On montrera dans la suite qu'il est nécessaire de disposer d'un détecteur $(R)$ résolu dans deux directions de l'espace afin de pouvoir mesurer quantitativement la diffraction de surface. 


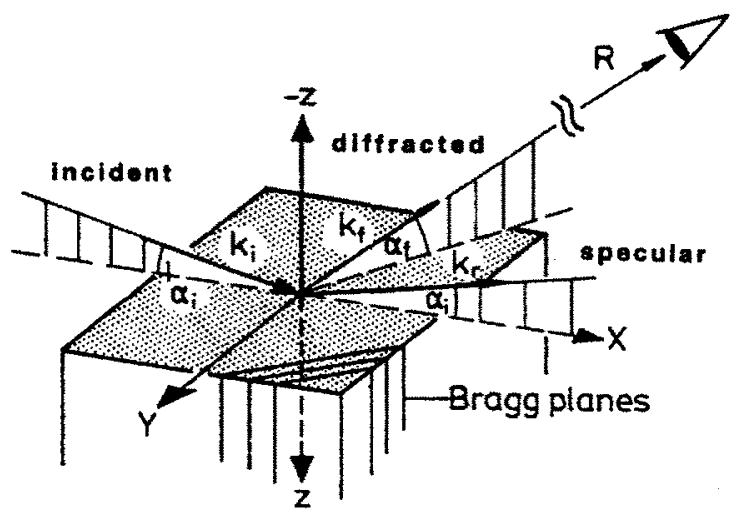

Figure 5 : géométrie de diffraction de surface (d'après Dosch [2])

\section{SPECIFICITE DES MESURES EN INCIDENCE RASANTE}
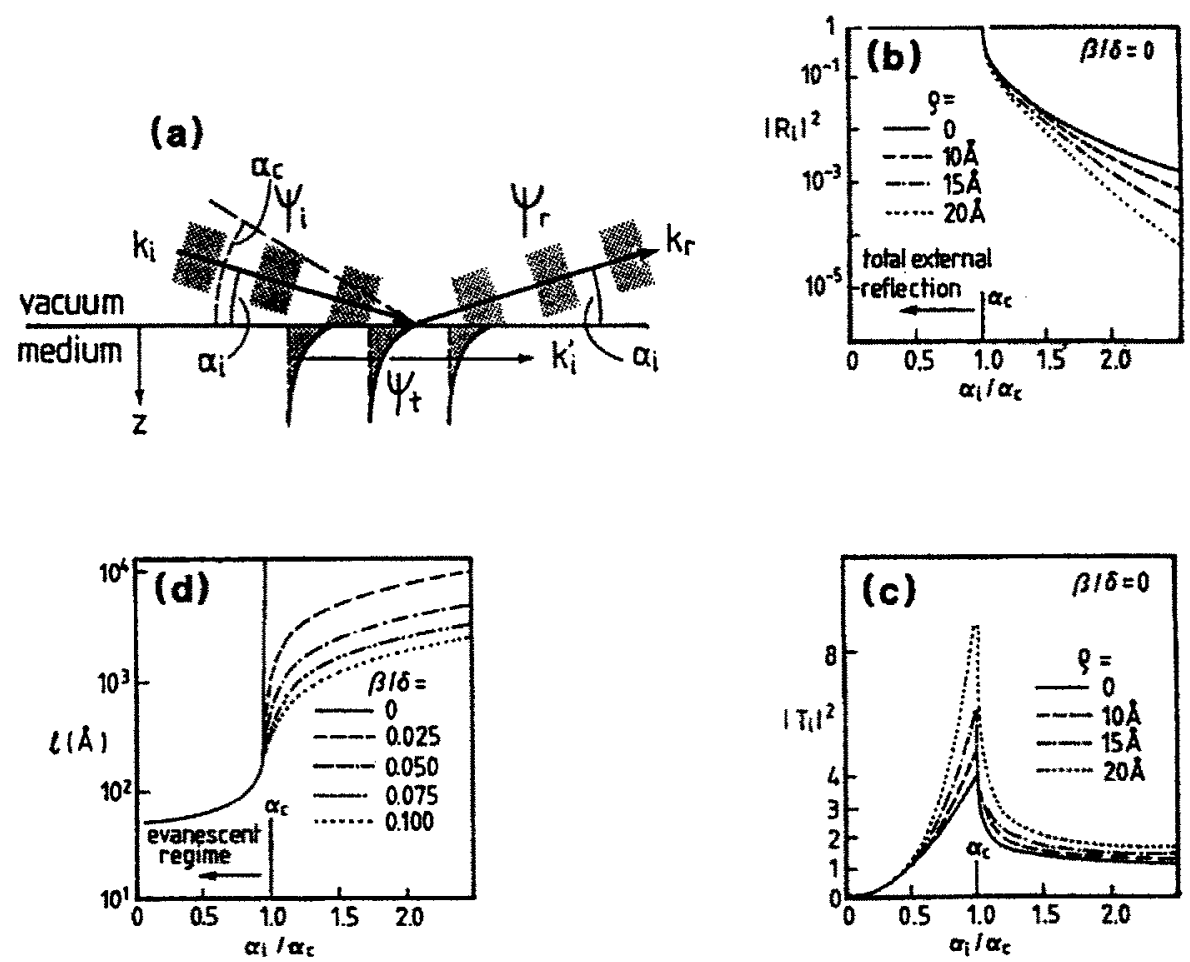

Figure 6: (a) onde incidente $\psi_{i}$ sur une couche mince; l'onde transmise $\psi_{i}$ est localisée à la surface. (c) amplitude de l'onde transmise en fonction de l'angle d'incidence. (d) profondeur de pénétration l en fonction de l'angle d'incidence. (Dosch [2]). 
La grande spécificité des mesures en incidence rasante est due au fait que l'interaction avec la couche mince étudiée est considérablement augmentée. En effet, en incidence rasante, par effet de localisation de l'onde incidente en surface (sous la forme d'une onde évanescente), la totalité du faisceau incident sur l'échantillon participe à la diffraction. La sensibilité à la surface est ainsi augmentée d'un facteur $10^{2}$ à $10^{3}$ par rapport à la diffraction sur le matériau massif. C'est ce qui rend possible la diffraction sur des échantillons d'une dizaine de nanomètres d'épaisseur. La Figure $6 \mathrm{~d}$ montre la profondeur de pénétration de l'onde incidente en fonction de l'angle d'incidence. L'angle d'incidence «optimal » correspond à l'angle critique. Autour de cette position, l'onde est localisée en surface et le facteur $\left|T_{i}\right|^{2}$ présente aussi un maximum, ce qui donnera une amplitude d'onde diffractée plus importante.

L'interprétation des mesures de diffraction de surface est un peu plus délicate qu'une mesure SANS classique car il y a transfert de moment à la fois perpendiculairement à la couche et celui dû à la structure que l'on veut étudier.

Pour une discussion détaillée du formalisme, consulter les références suivantes : (Dosch [3]).

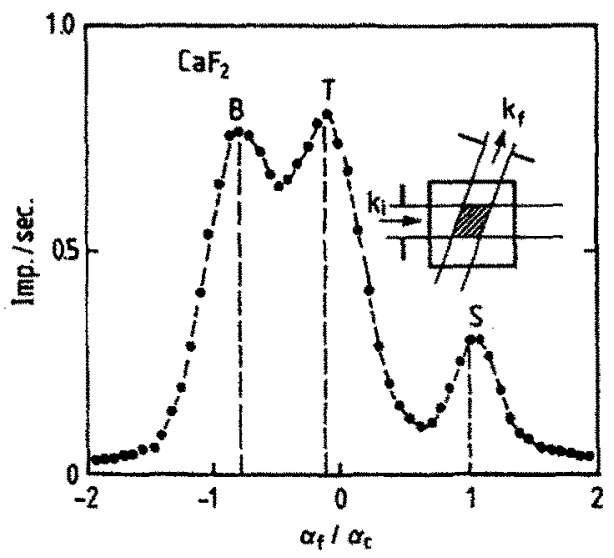

Figure 7: diffraction sur le pic de Bragg (111) d'un cristal de CaFe $e_{\dot{z}}(211)$ pour un angle d'incidence $\alpha_{i}=\alpha_{c}$ (Dosch (2).

Dans une expérience de diffusion spéculaire en incidence rasante, il faut être prudent car de nombreux signaux peuvent être mesurés et il est nécessaire de soigneusement les distinguer. La Figure 7 illustre ce point. Si on considère un faisceau en incidence rasante sur la surface d'un dioptre (ici un cristal de $\mathrm{CaFe}_{2}$ ), on constate que dans la direction de diffraction apparaissent plusieurs pics. Le premier (B) est dû à la diffraction dans le "bulk" du matériau. L'origine de ces neutrons dans la partie massive du matériau est que tous les neutrons ne sont pas réfléchis par la surface mais une partie d'entre eux (pour des raisons expérimentales) rentre par le "coté" de l'échantillon et est simplement diffractée. On observe un pic (T) qui correspond à la fraction de neutrons qui est transmise à travers la surface (cette proportion dépend de l'angle d'incidence) et finalement on observe le pic de diffraction de surface proprement dit (S) qui correspond aux neutrons qui ont subi une diffraction mais qui sont restés localisés à la surface du dioptre. 


\section{TRAITEMENT THEORIQUE DE LA DIFFUSION HORS SPECULAIRE}

Les problèmes de rugosité de surface sont des problèmes cruciaux en réflectivité. Un effort théorique très important a été conduit ces quinze dernières années dans le domaine de la réflectivité $X$. Ces efforts sont transposables dans le domaine de la réflectivité de neutrons.

Dans ce paragraphe nous présenterons quelques aspects du formalisme de l'approximation de Bom et de l'approximation DWBA (Distorted Wave Born Approximation). Puis nous présenterons un formalisme de calcul dynamique.

\subsection{Approximation de Born}

\subsubsection{Calcul de la section efficace de diffusion dans l'approximation de Born (Sinha [4])}

Dans l'approximation de Born (voir cours d'introduction), la section efficace de diffusion (SED) est donnée par:

où

$$
\frac{d \sigma}{d \Omega}=\left|f\left(\mathbf{k}_{i}, \mathbf{k}_{o}\right)\right|^{2}
$$

$$
f\left(\mathbf{k}_{i}, \mathbf{k}_{o}\right)=\frac{m}{2 \pi \hbar^{2}} \int_{\Omega} d v V(\mathbf{r}) e^{-i\left(\mathbf{k}_{o}-\mathbf{k}_{i}\right) \cdot \mathbf{r}}=-\frac{m}{2 \pi \hbar^{2}} \tilde{V}(\mathbf{q})
$$

est l'amplitude de diffusion.

Considérons un dioptre plan constitué d'atomes ayant une longueur de diffusion $b$ et de densité atomique $N$. La section efficace de diffusion peut se mettre sous la forme explicite :

où $\mathbf{q}=\mathbf{k}_{0}-\mathbf{k}_{\mathrm{i}}$ est le vecteur de diffusion.

$$
\frac{d \sigma}{d \Omega}=N^{2} b^{2} \int_{V} d \mathbf{r} \int_{V} d \mathbf{r}^{+} e^{-i \mathbf{q}(\mathbf{r}-\mathbf{r})}
$$

Considérons une surface rugueuse (voir Figure 8). Il est possible d'intégrer l'équation ci-dessus sur le volume du dioptre (de $-\infty$ à $z(x, y)$ ).

Nota : pour s'assurer de la convergence en $-\infty$, il suffit d'introduire une petite absorption dans le milieu.

On obtient alors l'expression suivante :

$$
\frac{d \sigma}{d \Omega}=\frac{N^{2} b^{2}}{q_{z}^{2}} \iint_{S_{0}} d x d y \iint_{S_{0}} d x^{\prime} d y^{\prime} \exp \left(-i q_{z}\left(z(x, y)-z\left(x^{\prime} y^{\prime}\right)\right)\right) \exp \left(-i q_{x}\left(x-x^{\prime}\right)-i q_{y}\left(y-y^{\prime}\right)\right)
$$

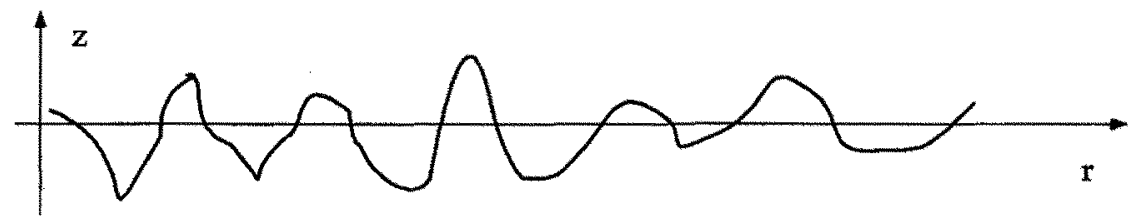

Figure 8 : profil d'une surface rugueuse.

La rugositê de la surface (voir Figure 8) est caractérisée par la distribution des hauteurs $z(x, y)$. La déviation standard $\sigma$ est définie par : 
On pose :

$$
\sigma=\sqrt{\left\langle z^{2}\right\rangle_{s}}
$$

$$
g(X, Y)=\left\langle\left[z\left(x^{*}, y^{\prime}\right)-z(x, y)\right]^{2}\right\rangle
$$

où la moyenne est prise sur toutes les paires de points de la surface.

Reprenons l'expression de la section efficace vue ci-avant. Remplaçons une des intégrations de surface par une moyenne d'ensemble (on pose $X=x-x^{\prime}$ et $Y=y-y^{\prime}$ ) :

$$
\frac{d \sigma}{d \Omega}=\frac{N^{2} b^{2}}{q_{z}^{2}} L_{x} L_{y} \iint_{S_{0}} d X d Y\left\langle\exp \left(-i q_{z}(z(X, Y)-z(0,0))\right)\right) \exp \left(-i q_{x} X-i q_{y} Y\right)
$$

Nota : cela suppose que la distribution des hauteurs est ergodique $: z(r)-z\left(\boldsymbol{r}^{\prime}\right)$ ne dépend que de $\boldsymbol{R}=$ $\boldsymbol{r}-\boldsymbol{r}$ '.

On peut utiliser un résultat des distributions gaussiennes (Mandel [5]) :

$$
\left\langle e^{i s u}\right\rangle=e^{-s^{2}\left\langle u^{2}\right\rangle / 2}
$$

D'où l'expression de la SED :

$$
\frac{d \sigma}{d \Omega}(\mathbf{q})=\frac{N^{2} b^{2}}{q_{z}^{2}} L_{x} L_{y} \iint_{S_{0}} d X d Y e^{g_{z}^{2} g(X, Y) / 2} e^{-i\left(q_{x} X+q_{y} Y\right)}
$$

Dans le cas de surfaces résultant de processus faisant intervenir un grand nombre d'événements aléatoires (arrivée d'atomes lors d'un dépôt sous vide par exemple), la distribution de probabilité $p(h)$ des hauteurs $z(r)$ peut être décrite par une distribution gaussienne (Ogilvy, 1991). Cependant, la distribution gaussienne est souvent seulement approchée (e.g. sur une surface polie, le polissage écrête les grands pics mais laisse les creux inchangés). Des études récentes introduisent des modèles de surface self-affines (fractales) qui sont invariantes par changement d'échelle. La fonction $g$ est alors décrite par une forme du type :

$$
g(\mathbf{R})=A R^{2 h},(0<h<1)
$$

La dimension de la surface est $D=3$ - h. Quand h est proche de 1 , la surface est peu rugueuse, quand $\mathrm{h}$ tend vers 0 , la surface devient très heurtée. Cependant l'équation ci-dessus décrit une surface idéale. La fonction $\mathbf{g}(\mathbf{R})$ diverge pour $R \rightarrow \infty$. En pratique, cette fonction sature à une valeur de $2 \sigma^{2}$. On peut alors la mettre sous la forme :

$$
g(\mathbf{R})=2 \sigma^{2}\left(1-e^{-(R / \xi)^{2 h}}\right), \quad(0<h<1)
$$

La longueur $\xi$ est la distance caractéristique au delà de laquelle la surface apparaît plane (distance de cut-off).

La fonction $g(\mathbf{R})$ peut se mettre sous la forme :

$$
g(X, Y)=2\left\langle z^{2}\right\rangle-2\langle z(X, Y) z(0,0)\rangle
$$

On définit la fonction d'autocorrélation des hauteurs $C(r)$ par :

$$
C(\mathbf{R})=\langle z(0) z(\mathbf{R})\rangle_{s}
$$

Qui peut se mettre sous la forme :

$$
C(\mathbf{R})=\left\langle z^{2}\right\rangle-\frac{1}{2} g(X, Y)=\sigma^{2} e^{-(R / \xi)^{2 n}}
$$

La SED devient :

$$
\left(\frac{d \sigma}{d \Omega}\right)(\mathfrak{q})=\frac{N^{2} b^{2}}{q_{z}^{2}} L_{x} L_{y} e^{-q_{z}^{z} \sigma^{2}} \iint_{S_{0}} d X d Y e^{q_{z}^{2} C(X, Y)} e^{-i\left(q_{x} X+q_{y} Y\right)}
$$

Cette expression peut se mettre sous la forme : 


$$
\left(\frac{d \sigma}{d \Omega}\right)_{1 o t}(\mathbf{q})=\left(\frac{d \sigma}{d \Omega}\right)_{s p e c}(\mathbf{q})+\left(\frac{d \sigma}{d \Omega}\right)_{d i f f}(\mathbf{q})
$$

avec

$$
\begin{gathered}
\left(\frac{d \sigma}{d \Omega}\right)_{s p e c}(\mathbf{q})=\frac{N^{2} b^{2}}{q_{z}^{2}} L_{x} L_{y} e^{-q_{z}^{2} \sigma^{2}} \delta\left(q_{x}\right) \delta\left(q_{y}\right) \\
\left(\frac{d \sigma}{d \Omega}\right)_{d i f f}(\mathbf{q})=\frac{N^{2} b^{2}}{q_{z}^{2}} L_{x} L_{y} e^{-q_{z}^{z} \sigma^{2}} \iint_{S_{0}} d X d Y\left(e^{q_{z}^{2} C(X, Y)}-1\right) e^{-i\left(q_{x} X+q_{y} Y\right)}
\end{gathered}
$$

où on met en évidence la diffusion purement spéculaire et la diffusion hors spéculaire.

L'intensité spéculaire est simplement l'intensité réfléchie sur la couche plane parfaite multipliée par un facteur d'atténuation $\exp \left(-q_{z}^{2} \sigma^{2}\right)$. On retrouve le résultat de Névot-Croce (facteur de DebyeWaller, voir cours précédent).

L'intensité diffusée non spéculaire mesure quant à elle le spectre des rugosités (transformée de Fourier de la fonction de corrélation des hauteurs $\mathrm{C}(\mathrm{X}, \mathrm{Y})$ )

Cependant, pour de petites valeurs de $q_{z}$, l'approximation de Born est invalide. En effet, en réflectivité, au niveau du plateau de réflectivité totale, l'ensemble de l'onde est diffusée. Cela est parfaitement contradictoire avec l'approximation de Born qui suppose que seule une petite fraction de l'onde est diffusée (voir cours d'introduction). En première approximation, on peut dire que l'approximation de Born devient applicable au-delà de $q_{z}>3 q_{c}$.

On peut pousser plus le loin le développement de Born et au lieu de s'arrêter à l'ordre 0 (correspondant à l'approx. de Born) développer à l'ordre 1. Ce développement est généralement appelé: Distorted Wave Born Approximation. II est relativement long de développer le calcul complet dans l'approximation DWBA. Nous renvoyons aux références suivantes pour un calcul détaillé (Messiah [6]; Sinha [4] ou Daillant [7], chap.4). Je rappelle simplement le principe.

Considérons à nouveau notre surface rugueuse (voir Figure 8).

Définissons le plan $z=0$ tel que;

$$
\iint_{S} d x d y z(x, y)=0
$$

On peut écrire le potentiel d'interaction sous la forme:

$$
V=V_{1}+V_{2}
$$

avec

$$
V_{1}=\left\{\begin{array}{cc}
k_{0}^{2},-a<z<0 \\
0, \quad z>0
\end{array} \text { et } V_{2}=\left\{\begin{array}{ccc}
k_{0}^{2}\left(1-n^{2}\right), & \text { pour } 0<z<z(x, y) \text { si } z(x, y)>0 \\
-k_{0}^{2}\left(1-n^{2}\right), & \text { pour } 0<z<z(x, y) \text { si } z(x, y)<0 \\
0, \text { ailleurs } &
\end{array}\right.\right.
$$

Le potentiel $V_{1}$ est le potentiel d'interaction avec la surface non rugueuse (idéale). L'interaction avec $\mathrm{V}_{1}$ est calculable exactement et cela nous permet de calculer les fonctions solutions de base $\psi_{1}$ (à l'ordre zéro). A ce potentiel se rajoute le potentiel de perturbation $\mathrm{V}_{2}$ qui décrit la rugosité de la surface.

Soit $\phi_{I}$, l'onde incidente. Soit $\psi_{1}$ la solution du problème avec l'interaction $V_{I}$ (surface non perturbée). Dans l'approximation DWBA, la diffusion entre les états $k_{i}$ et $k_{o}$ est donnée par:

$$
\left\langle k_{o}|V| k_{i}\right\rangle=\left\langle\psi_{1}^{*}\left|V_{1}\right| \phi_{1}\right\rangle+\left\langle\psi_{1}^{*}\left|V_{2}\right| \psi_{1}\right\rangle
$$

avec

$$
f\left(\mathbf{k}_{i}, \mathbf{k}_{o}\right)=-\frac{m}{2 \pi \hbar^{2}}\left\langle k_{o}|V| k_{i}\right\rangle
$$


Après un certain nombre de calculs (assez longs...), on peut montrer que:

$\operatorname{avec} S\left(\mathbf{q}_{t}\right)=\frac{\exp \left(-\left[\left(q_{z}^{t}\right)^{2}+\left(q_{z}^{*}\right)^{2}\right] \sigma^{2} / 2\right)}{\left|q_{z}^{t}\right|^{2}} \iint_{S} d X d Y\left(\exp \left(\left|q_{z}^{t}\right|^{2} C(X, Y)\right)-1\right) \exp \left(i\left(q_{x} X+q_{y} Y\right)\right)$

Cette forme de la SED appelle quelques remarques: (comparaison avec l'expression obtenue dans l'approximation de Born). En préfacteur apparaissent les termes $T\left(\mathbf{k}_{1}\right)$ et $T\left(\mathbf{k}_{2}\right)$. Ces termes ont une

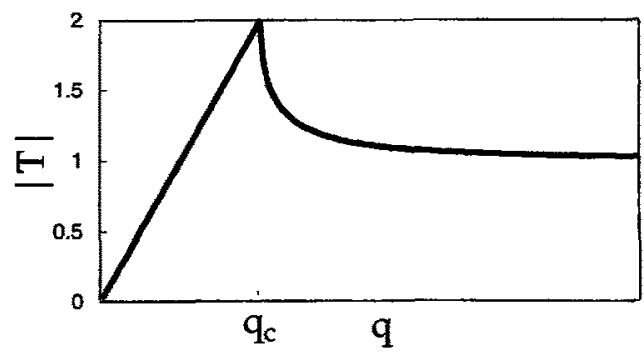

importance particulière étant donnée leur forme:

En effet, les coefficients $T$ présentent des maxima à la position $k=k_{c}$. Cela va donner naissance à des pics dans la diffusion hors spéculaire appelés "pics de Yoneda".

Dans le cas où $q_{z}^{t}$ est petit $\left(q_{z} \sigma<<1\right)$, l'expression de $S\left(q_{t}\right)$ peut se mettre sous la forme:

$$
S\left(\mathbf{q}_{t}\right)=\iint_{S} d X d Y C(X, Y) \exp \left(i\left(q_{x} X+q_{y} Y\right)\right)
$$

On voit que la diffusion hors spéculaire mesure dans ce cas la transformée de Fourier de la fonction de corrélation des hauteurs de la surface

La Figure 9 présente une série de scans en $q_{x}$ calculés dans diverses approximations (Born, DWBA, DWBA approché) pour différentes valeurs de la rugosité. On peut observer les pics de Yoneda de part et d'autre du pic spéculaire. Lorsque la rugosité augmente, l'intensité diffusée augmente au dépend de l'intensité réfléchie spéculairement. 


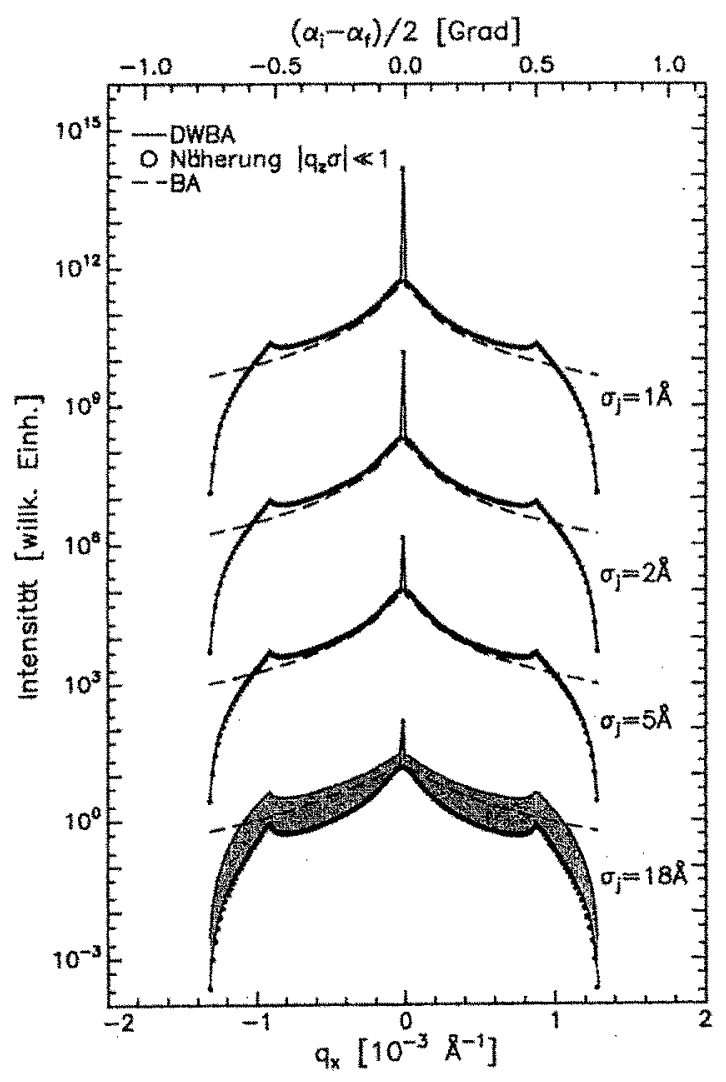

Figure 9: exemples de scans en $q_{x}$ pour des surfaces de rugosité croissante. Noter les pics de Yoneda autour des positions $q_{x}=10^{-3} A^{-1}$. Les intensités des différentes courbes ont été divisée par un facteur arbitraire pour rendre la figure plus lisible. D'après (Stettner [8]).

\subsubsection{Rugosité magnétique}

Dans le cas de couches minces magnétiques, il peut y avoir apparition de domaines magnétiques qui correspondent à des fluctuations de densité magnétique. Ces fluctuations doivent être traitées comme de la rugosité. La taille caractéristique des domaines se situe en général dans l'échelle de rugosité intermédiaire (cf. ci-dessus $0.5 \mu \mathrm{m}$ à $50 \mu \mathrm{m}$ ) et donne donc lieu à un signal de réflectivité non spéculaire.

Une difficulté de calcul apparaît. En effet, l'apparition de domaines magnétiques crée un champ magnétique à l'extérieur de la couche et complique beaucoup les calculs de réflectivité. Mais souvent la taille des domaines est très grande devant l'épaisseur des couches étudiées et le champ dipolaire extérieur à la couche peut être négligé.

\subsection{Formalisme dynamique dans le cas de structures lithographiées}

Comme il a été vu au cours précédent, dans un problème de réflectivité, où le potentiel d'interaction est uniquement fonction de $z$, il est possible de résoudre exactement l'équation de Schrödinger. Si 
l'on traite le problème d'un système multicouches, il est possible de subdiviser ce système multicouches en une superposition de couches dans lesquelles on résout l'équation de Schrödinger. Le calcul de la diffusion par le système complet peut ensuite être obtenu en ajustant les conditions aux limites des différentes fonctions d'ondes.

En réflectivité spéculaire on est donc essentiellement amené à résoudre l'équation:

$$
\left[\frac{-\hbar^{2}}{2 m} \Delta+V(z)\right] \varphi(\mathbf{r})=E \varphi(\mathbf{r})
$$

où le potentiel d'interaction est une fonction de $z$ uniquement.

Dans le cas d'un système périodique tel un réseau, le potentiel est une fonction à la fois de $z$ mais aussi de $x$. L'équation à résoudre est donc:

$$
\left[\frac{-\hbar^{2}}{2 m} \Delta+V(x, z)\right] \varphi(\mathbf{r})=E \varphi(\mathbf{r})
$$

où $V$ est une fonction périodique de $x: V(z, x+d)=V(z, x)$.

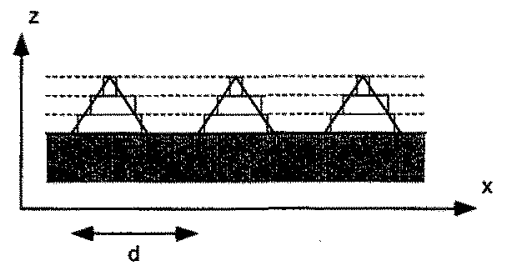

Figure 10: discrétisation en plusieurs couche d'un motif périodique en forme de pyramides.

On peut utiliser le même principe de calcul que celui qui a été utilisé dans la résolution du problème à une dimension. Le système est discrétisé suivant la direction $(\mathrm{Oz})$ de telle sorte que dans chacune des couches le potentiel ne soit plus qu'une fonction de $x$ (voir Figure 10). On est alors confronté à la résolution de l'équation suivante:

$$
\left[\frac{-\hbar^{2}}{2 m} \Delta+V(x)\right] \varphi(\mathbf{r})=E \varphi(\mathbf{r})
$$

où $V$ est une fonction périodique de $x: V(z, x+d)=V(z, x)$.

Transformons cette équation en équation de Helmholtz en faisant apparaître le vecteur d'onde et lindice optique :

$$
\Delta \varphi+k_{0}^{2} n^{2}(x) \varphi=0
$$

où $n(x)$, lindice optique est une fonction périodique : $n(x+d)=n(x)$.

Il est possible de résoudre cette équation en cherchant des solutions sous la forme:

$$
\varphi(x, z)=\sum_{n} \varphi_{n}(z) e^{i \frac{2 \pi}{d} x} \text { et en posant } k^{2}(x)=\sum_{n} c_{n} e^{i n \frac{2 \pi}{d} x}
$$

On obtient alors une équation vectorielle qui peut se mettre sous la forme d'une équation aux valeurs propres:

$$
-k_{\perp}^{2} \Phi=\mathrm{V} \Phi
$$

dont les valeurs propres et les vecteurs propres correspondent respectivement aux vecteurs d'onde propres et aux fonctions d'onde propres se propageant dans la tranche de matériau considérée. C'est à dire que l'on obtient les modes propres de propagation dans la tranche de matériau mais l'amplitude de ces différents modes les uns par rapport aux autres n'est pas connue.

Pour déterminer les amplitudes des différents modes, il faut appliquer les conditions aux limites aux interfaces entre les différentes tranches. A l'interface d'une tranche $i$ et d'une tranche $i+1$, on obtient une relation reliant les amplitudes des différents modes: 


$$
D_{i}\left[\begin{array}{l}
{[u]_{i}} \\
{[d]}
\end{array}\right]=D_{i+1}\left[\begin{array}{l}
{[u]_{i+1}} \\
{[d]_{i+1}}
\end{array}\right]
$$

où $D$ sont les matrices caractérisant les conditions aux interfaces et $[u]$ te $[d]$ sont les vecteurs des amplitudes des différents modes de propagation.

La résolution du problème complet (détermination des amplitudes des différents modes) est obtenue par récurrence (de type Parrat). Ce formalisme est tout à fait similaire à ce qui est utilisé pour le calcul matriciel de la réflectivité à la seule exception que dans chaque tranche on n'a pas affaire à 2 modes (up et down) mais à $2 \times \mathrm{n}$ modes. Ce formalisme peut-être étendu au cas magnétique.

\section{UTILISATION PRATIQUE DES REFLECTOMETRES EN DIFFUSION HORS SPECULAIRE.}

Dans ce paragraphe nous allons décrire les différentes techniques expérimentales de mesures hors spéculaire en réflectivité de neutrons. Nous séparerons le cas des spectromètres à longueur d'onde fixe et le cas des spectromètres en temps de vol.

\subsection{Les différents modes de mesure sur un spectromètre deux axes}

Sur un réflectomètre deux axes, il est possible de se déplacer dans le plan $\left(q_{x}, q_{z}\right)$ de l'espace réciproque. Les directions de scan privilégiées sont bien sûr les directions $q_{z}=c s t e$ et $q_{x}=c s t e$. La Figure 11 décrit les différents modes de scan possibles.

Un $\operatorname{scan} \theta / 2 \theta$ est un scan suivant la direction $q_{z}$. C'est le type de mesure qui est utilisé dans les mesures classiques de réflectivité spéculaire. La mesure de l'intensité du signal de réflectivité en fonction de $q_{z}$ (ou de $\theta_{i}$ ) permet de sonder la structure de l'échantillon suivant l'axe $(\mathrm{Oz})$ et de déterminer sa structure chimique et magnétique suivant $(\mathrm{Oz})$ comme cela a été décrit ci-avant.

a) Rocking curve

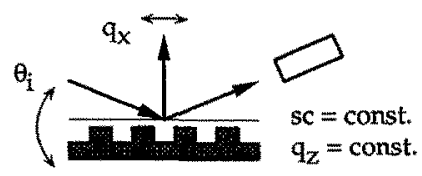

b) Bragg scan

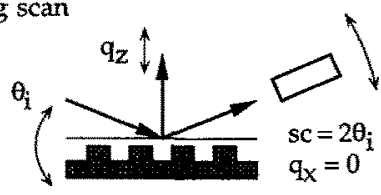

c) Scan détecteur.

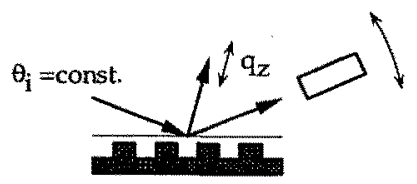

Figure 11 : les différents modes de mesure possibles sur un spectromètre deux axes. (a) rocking curve: $l$ 'angle $\theta$ est balayé, $2 \theta$ est fixe ; (b) scan $\theta / 2 \theta$ classique; (c) scan détecteur : seul l'angle $2 \theta$ est balayé. (adapté de (Tolan 1993)) 
La deuxième direction de scan dans l'espace réciproque est la direction $q_{x}$. Ce type de balayage est obtenu par une rotation de type « rocking curve » (Figure 11a) : la position du faisceau incident est maintenue constante par rapport au détecteur et l'échantillon seul subit une rotation. Ce type de mesure donne des renseignements sur la structure planaire de la couche et est donc le type de mesure privilégié lors d'études de diffusion non spéculaire et de structures lithographiées. Mécaniquement, on peut envisager un autre type de scan qui consiste en une rotation du détecteur. Cependant ce type de scan suit une trajectoire non triviale dans l'espace réciproque et ne donne pas de manière directe des informations physiques. Il est en général limité à des fins de réglages. La Figure 12 illustre les trajectoires dans l'espace réciproque pour les trois modes de mesure présentés sur la Figure 11. On notera que dans une mesure de type rocking curve, la courbure de la trajectoire est imperceptible (dans le domaine de $q_{x}$ exploitable).

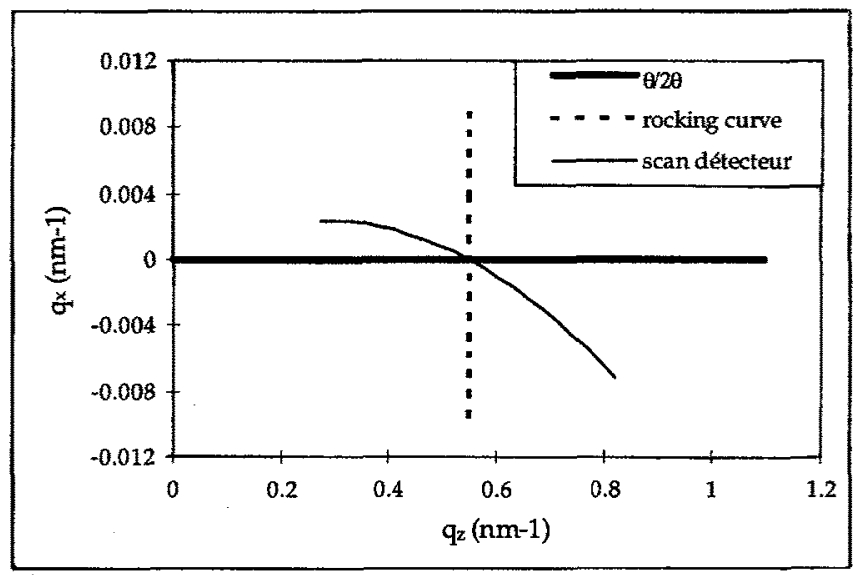

Figure 12 : trajectoires dans l'espace réciproque $(q z, q x)$ pour différents modes de mesure: scan $\theta / 2 \theta$ jusqu'à $2^{\circ}$, rocking curve autour de $1^{\circ}\left(\right.$ de $\left.-1 \grave{a}+1^{\circ}\right)$ et scan détecteur de $0^{\circ} \grave{a} 2^{\circ}$ (incidence $\left.1^{\circ}\right)$.

\subsection{Les différents modes de mesure sur un spectromètre en temps de vol}

Sur un spectromètre en temps de vol, le scan spéculaire classique $2 \theta / \theta$ est remplacé par une mesure à angles d'incidence et de réflexion fixes et égaux. La longueur d'onde des neutrons varie (typiquement dans la gamme $0.3 \mathrm{à} 3 \mathrm{~nm}$ ) et permet de balayer l'espace réciproque suivant $q_{z}$.

Lors d'une mesure de type rocking curve (détecteur fixe, angle $\theta$ de l'échantillon variable), les trajectoires dans l'espace réciproque sont très différentes des trajectoires obtenues sur un spectromètre 2 axes. La Figure 13a montre les trajectoires parcourues dans une mesure de type rocking curve. Le plan $(q z, q x)$ est parcouru par des trajectoires formant une figure en éventail. Chaque trajectoire est constituée des mesures obtenues pour des neutrons de longueur d'onde variant de $0.3 \mathrm{~nm}$ à $2.5 \mathrm{~nm}$. Dans une mesure de type scan détecteur (Figure 13b) les trajectoires deviennent asymétriques par rapport à $q_{x}=0$. Sur un spectromètre en temps de vol, il n'est pas possible de suivre directement une trajectoire à $q_{z}$ constant, il faut la reconstruire numériquement. 

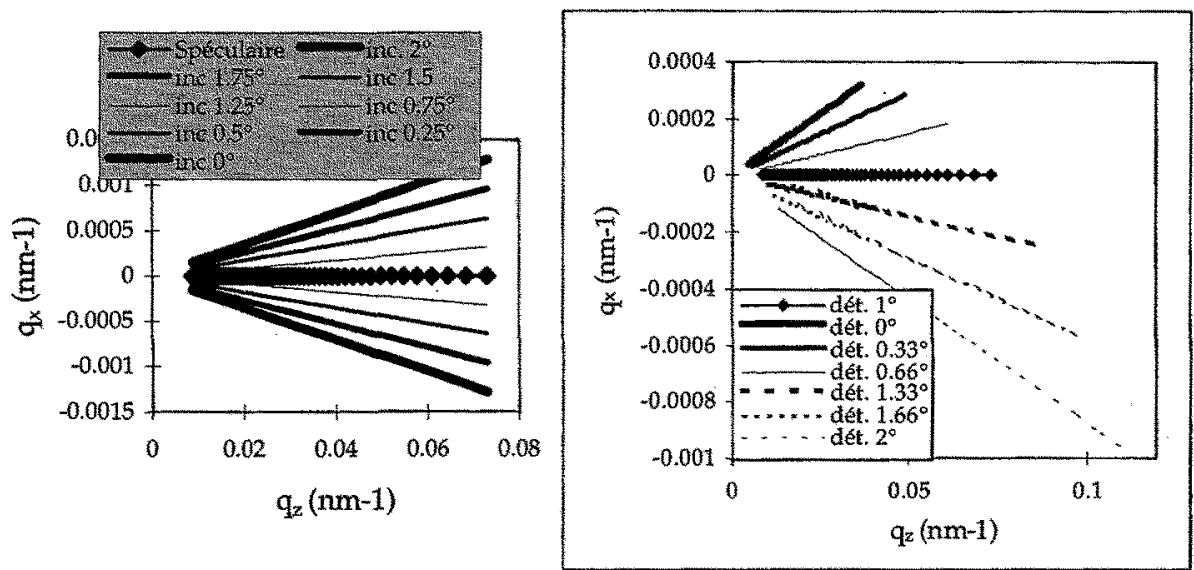

Figure 13 : trajectoines dans l'espace réciproque sur un spectromètre en temps de vol en fonction de la longueur $d^{\prime}$ onde (de $0.3 \mathrm{~nm}$ d $2.5 \mathrm{~nm}$ ). A gauche, rocking curves autour de $1^{\circ}$. A droite, scan détecteur di incidence fixe de $1^{\circ}$.

\subsubsection{Fonction de rếsolution du spectromètre EROS}

La forme de la fonction de résolution d'un spectromètre en temps de vol est :

$$
\left(\frac{\Delta q}{q}\right)^{2}=\left(\frac{\Delta \vartheta}{\vartheta}\right)^{2}+\left(\frac{\Delta \lambda}{\lambda}\right)^{2}
$$

A la différence du spectromètre à longueur d'onde fixe PADA, il est possible d'ajuster la résolution en longueur d'onde sur le spectromètre en temps de vol EROS. La résolution angulaire $\theta$ peut être ajustée entre $7 \times 10^{-3}$ à $7 \times 10^{-2}$ degrés. La résolution en longueur d'onde $\lambda$ peut être ajustée entre $0.015 \mathrm{~nm}$ et $0.15 \mathrm{~nm}$. Le hacheur simple disque du spectromètre EROS produisant des paquets de neutrons de largeur temporelle fixe, la résolution n'est pas constante sur tout le domaine $q_{z}$. Aux grandes longueurs d'onde, la résolution est dominée par la résolution angulaire tandis qu'aux courtes longueurs d'onde la résolution est dominée par la résolution en longueur d'onde. La Figure 14 montre la résolution en $q_{z}$ pour des conditions typiques $\left(\Delta \theta=0.02^{\circ}\right.$ et $\left.\Delta \lambda=0.02 \mathrm{~nm}\right)$ en fonction de la longueur d'onde et de l'angle d'incidence.

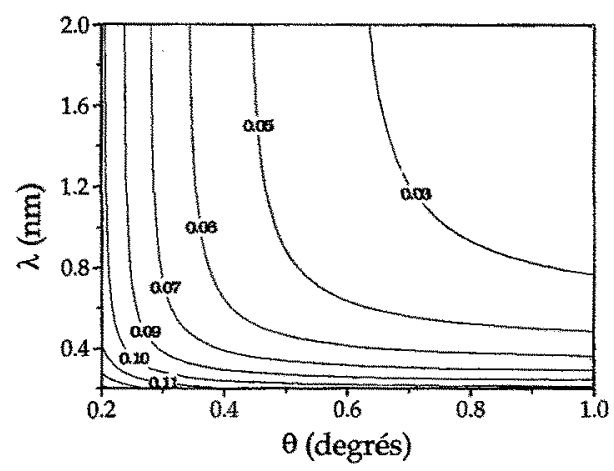

Figwre $14:$ résolution $\Delta q_{z} / q_{z}$ du spectromètre en temps de vol EROS en fonction de l'angle d'incidence et de la longueut f'onde (pour $\Delta \theta=0.02^{\circ}$ et $\Delta \lambda=0.02 \mathrm{~nm}$ ). 


\section{EXEMPLES DE DIFFUSION HORS SPECULAIRE SUR DES SYSTEMES MAGNETIQUES}

\subsection{Exemples de diffusion sur des réseaux périodiques lithographiés}

Dans ce paragraphe, nous allons présenter des mesures de diffusion hors spéculaire sur des systèmes modèles de type réseau de diffraction. Les mesures sur ce type de système présentent plusieurs avantages: le premier est que les intensités diffusées sont relativement importantes, le deuxième est quil est possible de traiter quantitativement cette diffusion. La figure ci-dessous montre les intensités diffusés dans l'espace réciproque mesurées sur un réseau de lignes de nickel déposées sur un substrat de verre.

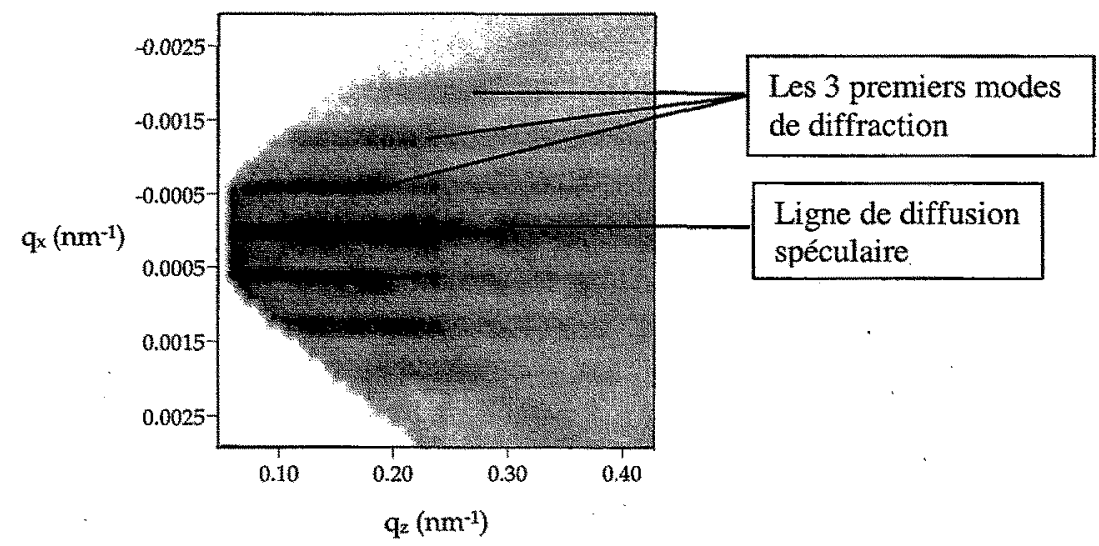

Figure 15: intensités diffusées par un réseau de lignes de nickel déposées sur un substrat de verre (périodicité 10 um, largeur $4 \mu \mathrm{m})$ (ntesure en neutrons non polarisés)

Les caractéristiques de la Figure 15 sont les suivantes. Le plan ( $q x, q z)$ présente deux zones triangulaires "grisées » (pour les petits $\mathrm{q}_{\mathrm{z}}$ et les grands $\mathrm{q}_{\mathrm{x}}$ ). Ces zones représentent les parties de l'espace réciproque inaccessibles pour raisons géométriques (voir Figure 13a). Dans la zone mesurable on observe une série de lignes pour lesquelles où l'intensité est non négligeable. La première, située autour de l'axe $q_{x}=0$, représente la diffusion spéculaire sur l'échantillon. Une coupe suivant $q_{x}=0$ donne la courbe de réflectivité spéculaire de l'échantillon. On observe par ailleurs deux ou trois autres lignes de par et d'autre de $q_{x}=0$ qui représentent les pics de diffusion non spéculaire. Ces zones suivent les lignes $\mathrm{q}_{\mathrm{x}}=m \times 0.0006 \mathrm{~nm}^{-1}$ qui correspondent à une période de $6 \mu \mathrm{m}\left(2 \pi / 10 \mu m=0.00063 \mathrm{~nm}^{-1}\right)$.

Nota: dans cet exemple, nous n'avons pas tenu compte du contraste magnétique (bien qu'il s'agisse de lignes de nickel).

Les réseaux lithographiés de matériaux magnétiques peuvent servir de systèmes modèles à l'étude de la diffusion hors spéculaire sur des systèmes magnétiques. Les structures réalisées peuvent couvrir les tailles entre $100 \mathrm{~nm}$ et $10 \mu \mathrm{m}$. Le comportement magnétique de réseaux de nanostructures a été étudié par C. Miramond au Service de Physique de l'Etat Condensé du Centre d'Etudes de Saclay (Miramond, 1997). 
S'ils sont suffisamment proches, les différents nanoeléments sont couplés magnétiquement entre eux par couplage magnétique dipolaire. En théorie, la configuration magnétique énergétiquement la plus favorable est un couplage antiferromagnétique. La Figure 16 illustre l'ordre magnétique dans le cas de quelques structures simples.

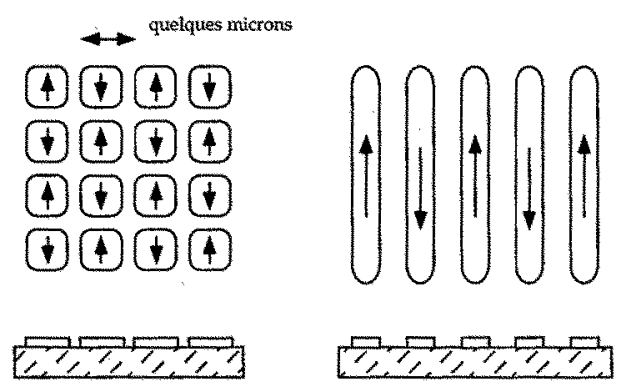

Figure 16: exemples de configurations magnétiques possibles dans des nanostructures de matériaux magnétiques. Les différents nanoéléments sont couplès magnétiquement entre eux par couplage dipolaire. La configuration magnétique énergétiquement la plus favorable est un couplage antiferronagnétique.

Cependant, en pratique les nanostructures magnétiques ne deviennent monodomaines que pour des tailles inférieures à environ $100 \mathrm{~nm}$ dans le cas d'alliages nickel-fer. Ces tailles varient en fonction du matériau, de l'épaisseur de la nanostructure ou du fait que l'aimantation est planaire ou perpendiculaire. Au-delà de ces tailles, des structures en domaines commencent à apparaître (voir Figure 17). Les illustrations de la Figure 16 ne peuvent représenter la réalité que dans le cas de motifs à aimantation perpendiculaire ou bien dans le cas de motifs très petits (quelques $100 \mathrm{~nm}$ ) dans le cas d'une aimantation planaire.

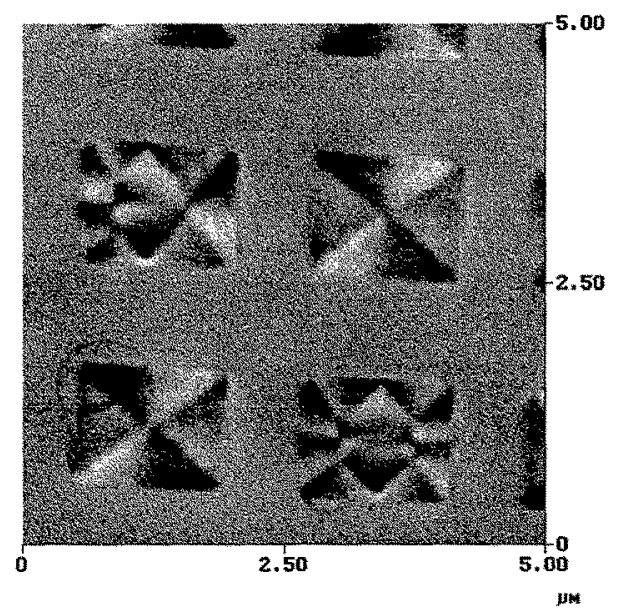

Figure 17: configuration magnétique interne de plots de permalloy observée par microscope à force magnétique (Warin, CEA/Saclay).

Si on suppose que l'on a effectivement un tel réseau de lignes couplées antiferromagnétiquement comme représenté sur la Figure 16, la diffraction sur un tel réseau doit faire apparaitre des pics de diffraction aux positions correspondants à la périodicité des motifs. De plus, les neutrons étant 
sensibles au contraste magnétique, des pics satellites supplémentaires de super-périodicité doivent apparaître aux positions correspondant à une périodicité double de la périodicité structurale du réseau. Dans le paragraphe suivant, nous allons essayer d'illustrer ces points.

Nous présentons des mesures sur des réseaux de lignes d'alliage nickel-fer (Ni $88 \%$ / $\mathrm{Fe} 12 \%$ ) déposées sur verre. La surface gravée des échantillons est de $1 \mathrm{~cm}^{2}$. Les largeurs typiques des lignes sont de 1 à quelques microns et l'espacement est du même ordre. L'épaisseur des lignes est de 640 $\mathrm{nm}$. Dans le cas de lignes fines très rapprochées, ce réseau magnétique s'ordonne de manière antiferromagnétique par couplage dipolaire entre les lignes comme décrit précédemment. Nos études de diffusion non spéculaire en neutrons ont été limitées à l'étude de réseaux fabriqués par lithographie optique car ceux-ci peuvent couvrir des surfaces de l'ordre du centimètre carré et sont donc exploitables en diffusion de neutrons. En revanche il n'est pas possible d'étudier des structures dont la périodicité est inférieure à $1 \mu \mathrm{m}$ environ et ceci pour deux raisons :

- ces structures sont réalisées par lithographie X ou par lithographie électronique et les surfaces d'échantillon sont très limitées $\left(1 \mathrm{~mm}^{2}\right.$ dans le cas de la lithographie $\mathrm{X}$ et $100 \times 100 \mu \mathrm{m}^{2}$ dans le cas de la lithographie électronique).

- la périodicité de ces structures est telle qu'il est nécessaire de faire des mesures à grand vecteur de diffusion $q_{\mathrm{x}}$. Ceci entraîne des limitations liées au flux de neutrons disponible.

Les échantillons de lignes magnétiques présentés ci-après ont été fabriqués par C. Miramond du SPEC

La Figure 18 montre le début d'une courbe de réflectivité sur un échantillon de lignes de permalloy déposées sur un substrat de silicium (périodicité $7 \mu \mathrm{m}$; largeur des lignes $5 \mu \mathrm{m}$ ). On peut constater que cet échantillon présente deux plateaux de réflectivité. La première coupure a lieu autour de $0.2^{\circ}$, ce qui correspond à l'angle critique du silicium. La deuxième coupure a lieu autour de $0.4^{\circ}$, ce qui correspond à l'angle critique du nickel. Si on normalise le plateau de réflectivité totale à 1 , on constate que l'intensité du deuxième plateau de réflectivité correspond à 0.4 environ. Cette valeur est loin de la valeur de $70 \%$ qui correspondrait au rapport des surfaces des lignes sur la surface du substrat. Numériquement, il n'est pas possible de rendre compte de l'apparition de ces deux plateaux, une telle structure apparaît seulement pour des taux de recouvrement faibles (environ $30 \%$ ). On en déduit donc que le code numérique ne permet pas de simuler de manière très satisfaisante un système où les lignes ont été inclinées pour ajuster la période effective.

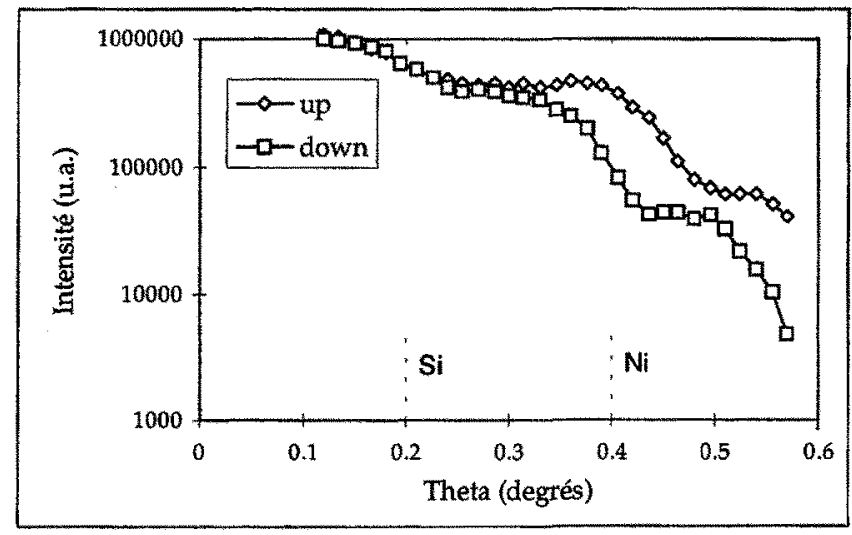

Figure 18: rêflectivité d'un réseau de lignes de NiFe de période 7 um et de largeur 5 pm. 
Le champ coercitif de lignes magnétiques dépend beaucoup de leur largeur. Pour des lignes de $5 \mu \mathrm{m}$ de large, le champ coercitif est de $14 \mathrm{G}$. Dans le cas de lignes de $1 \mu \mathrm{m}$, le champ coercitif augmente à une valeur de $60 \mathrm{G}$.

$N . B$. : dans le cas de nanostructures, le champ coercitif est a priori ambigu car il existe un champ coercitif lié à la structure magnétique interne des lignes et un champ «coercitif » lié à la forme et au couplage entre structures. On définit le champ coercitif comme le point où l'aimantation est nulle.

La Figure 19 montre une mesure de diffusion non spéculaire sur un réseau de lignes de permalloy (périodicité de $6 \mu \mathrm{m}$, largeur des lignes de $5 \mu \mathrm{m}$ ). Lors de la mesure, l'échantillon était incliné de $30^{\circ}$ ce qui donne une période effective vue par les neutrons de $12 \mu \mathrm{m}$. La Figure 19 montre une rocking curve autour de l'angle d'incidence $0.4^{\circ}$ pour deux états magnétiques différents. Les courbes sont tracées en échelle logarithmique pour rendre la mesure plus lisible. En effet, les intensités diffractées ne représentent environ que $1 \%$ du signal spéculaire. La diffraction par un motif de périodicité $12 \mu \mathrm{m}$ correspond à une position $\mathrm{q}_{\mathrm{x}}=0.52 \mu \mathrm{m}^{-1}$. On constate que cette valeur est en très bon accord avec les positions des premiers pics satellites de la Figure 19.

Sur la Figure 19a, un champ de $35 \mathrm{G}$ est appliqué. Ce champ est suffisant pour saturer magnétiquement ces lignes de $5 \mu \mathrm{m}$. On observe un grand signal magnétique sur le pic spéculaire (ce qui correspond à la différence d'indice «classique » $b_{+M}$ et $b_{-M}$ ). Au contraire, les intensités « up-up » et «down-down» se superposent parfaitement sur les pics satellites (correspondant à la périodicité du réseau). Cela est très surprenant si l'on exclut une simple coïncidence.

Le champ magnétique appliqué sur l'échantillon a ensuite été varié de $+\mathrm{H}_{\text {sat }}$ à $-5 \mathrm{G}$, afin de se placer dans un état où les lignes sont partiellement démagnétisées. L' « inversion » des courbes $(++)$ et $(-$ ) sur la Figure 19b est simplement due au fait que le champ appliqué sur l'échantillon est négatif. Sous le champ de $-5 \mathrm{G}$, on observe plusieurs différences qualitatives. Dans le pic spéculaire, la différence d'intensité entre «up-up » et « down-down » est plus faible ; cela est simplement due au fait que l'aimantation "globale » du système est plus faible sous $-5 \mathrm{G}$ que sous $35 \mathrm{G}$. Par ailleurs, aux positions intermédiaires $\mathrm{q}_{\mathrm{x}}= \pm 0.25 \mu \mathrm{m}^{-1}$, il y a eu un changement notable. Dans le cas saturé (Figure 19 gauche), on observe une différence entre les signaux « up-up » et «down-down». Au contraire, dans le cas partiellement démagnétisé, les courbes se superposent parfaitement à ces positions.
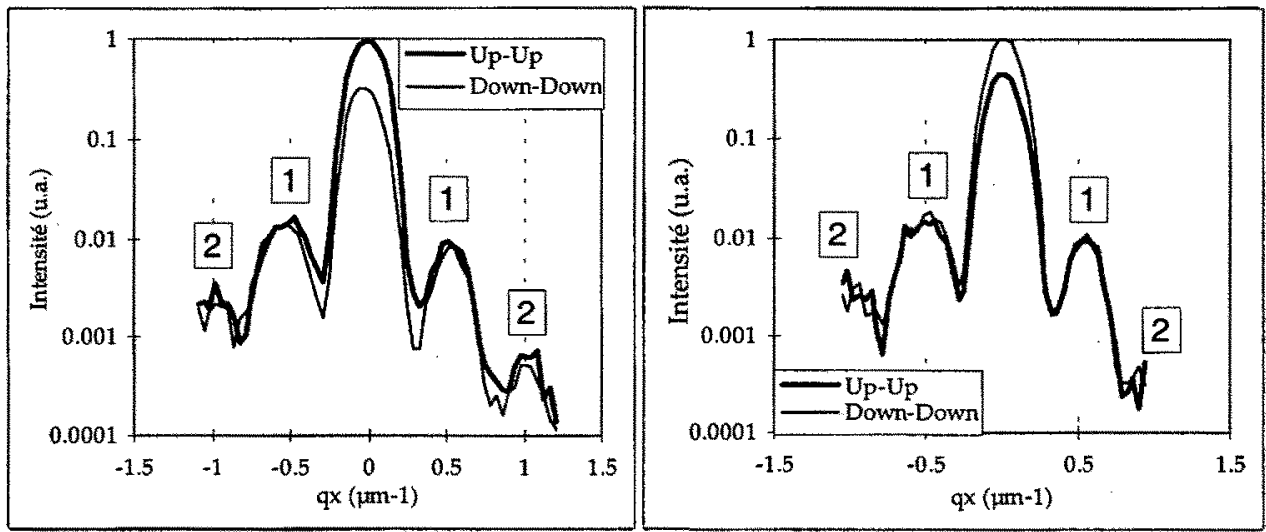

Figure 19: rocking curve autour de $0.4^{\circ}$ sur un réseau de période 6 um (lignes de 5um de large). A gauche sous un champ de $35 G ; \dot{t}$ droite sous un champ de $-5 G$. Noter l'inversion up-up et down-down. Les pics de diffraction sont indexês. 
La Figure 20 montre une mesure exactement identique à la mesure précédente à la seule différence qu'il s'agit de la rocking curve autour de $0.6^{\circ}$. On observe des changements qualitatifs très importants. La position des pics satellites principaux est maintenant autour de $q_{x}=1 \mu \mathrm{m}^{-1}$. Il existe cependant toujours un signal autour de la position $0.5 \mu \mathrm{m}^{-1}$.
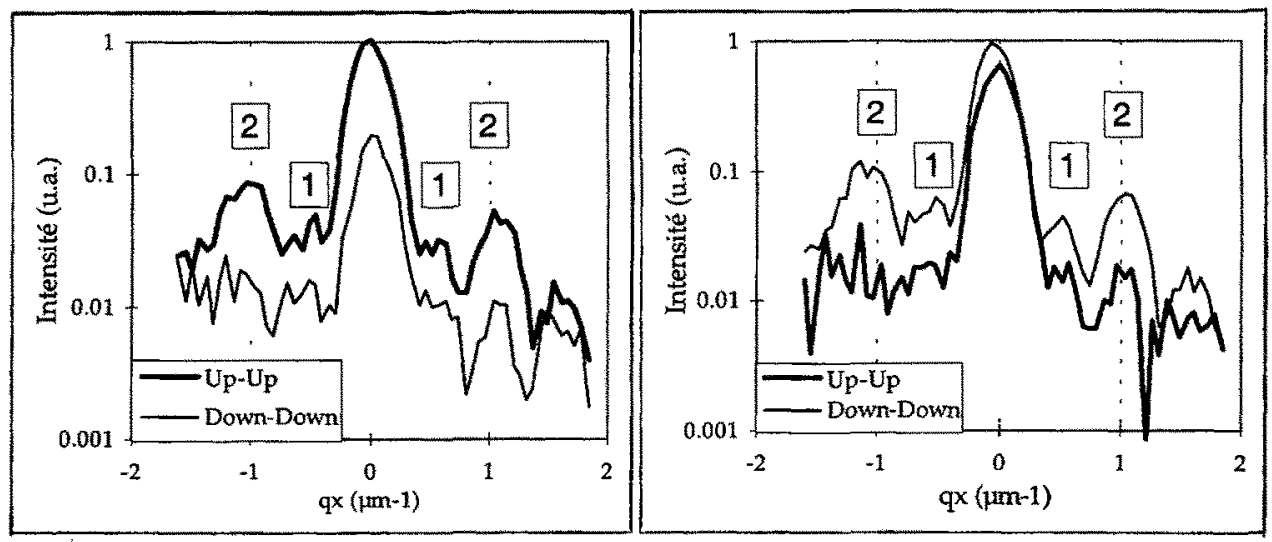

Figure 20: rocking curve autour de $0.6^{\circ}$ sur un réseau de période 6 um (lignes de $5 \mu m$ de large). A gauche sous un champ de $35 \mathrm{G}$; à droite sous un champ de $-5 \mathrm{G}$. Les pics de diffraction sont indexés. Noter l'inversion up-up et downdown.

Pour vérifier qu'il est effectivement possible d'obtenir des pics de diffraction d'ordre 2 d'intensité supérieure à l'intensité des pics d'ordre 1 , nous avons calculé numériquement les intensités diffractées sur un réseau de lignes de $\mathrm{NiFe}$ de période $6 \mu \mathrm{m}$. Nous avons supposé que le profil du réseau de lignes est rectangulaire. (lignes de largeur $5 \mu \mathrm{m}$ et de hauteur $650 \mathrm{~nm}$ ). L'intensité des modes de diffraction 0,1 et 2 est tracé en fonction de l'angle de diffusion $2 \theta$ (dans le cas d'une polarisation « up ») sur la Figure 21.

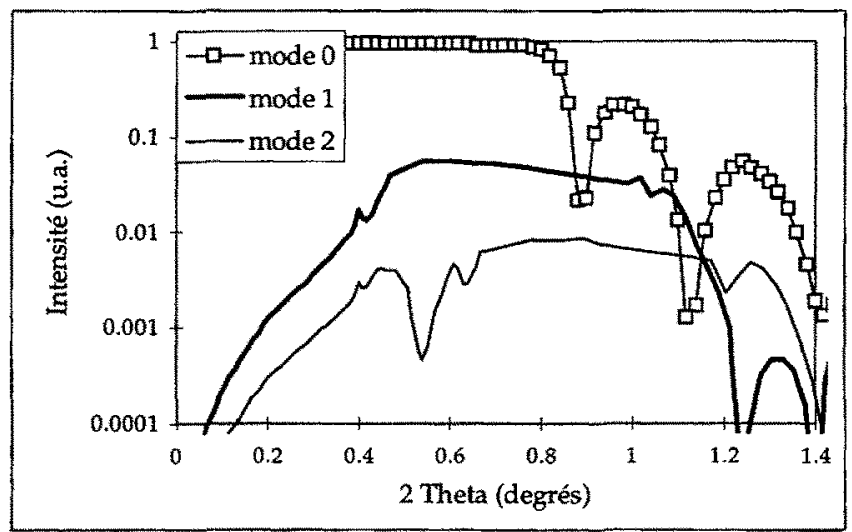

Figure 21 : simulation des intensités des modes diffractés en fonction de l'angle de mesure $2 \theta$ pour un réseau de périodicité $6 \mu m$ (lignes de NiFe de largeur 5 um et de hauteur $650 \mathrm{~nm}$ ) (Cas d'une polarisation *up »). 


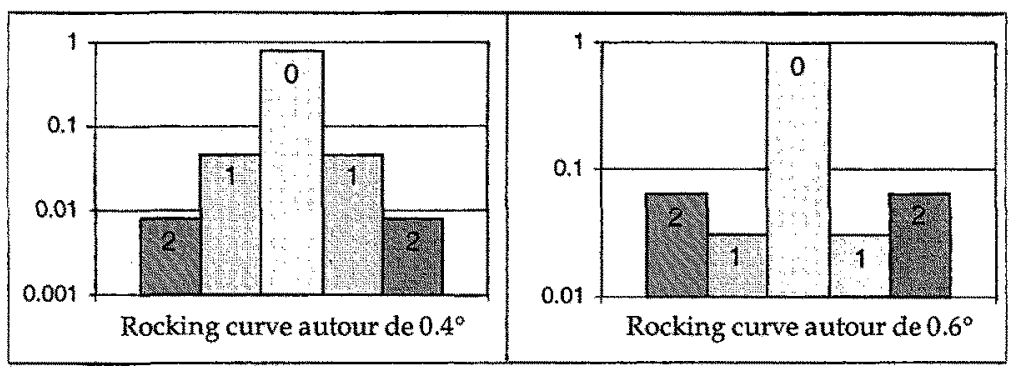

Figure 22 : intensités relatives des modes 0,1 et 2 pour des rocking curves autour de $0.4^{\circ}$ et de $0.6^{\circ}$ déduites des calculs de la Figure 21. Ces résultats se comparent qualitativement avec les mesures des Figure 19a et Figure $20 a$.

La Figure 22 résume les intensités relatives des différents modes 0,1 et 2 pour les deux rocking curves autour de $0.4^{\circ}$ et de $0.6^{\circ}$. Dans le cas de la rocking curve autour de $0.4^{\circ}$, on surestime les intensités des modes diffusés (voir Figure 19a). Dans le cas de la rocking curve autour de $0.6^{\circ}$, on observe bien un signal du mode +2 supérieur à celui du mode 1 . De plus les intensités sont bien reproduites quantitativement (voir Figure 20a). Evidemment, le calcul numérique ne permet pas de rendre compte de la forme des pics.

Nous avons ensuite étudié la diffusion un réseau de lignes de périodicité $5 \mu \mathrm{m}$ mais dont la largeur des lignes est réduite à $1 \mu \mathrm{m}$. On se rapproche du cas de lignes monodomaines. La Figure 23 montre une mesure de rocking curve autour de $0.5^{\circ}$ sur ce réseau de lignes.

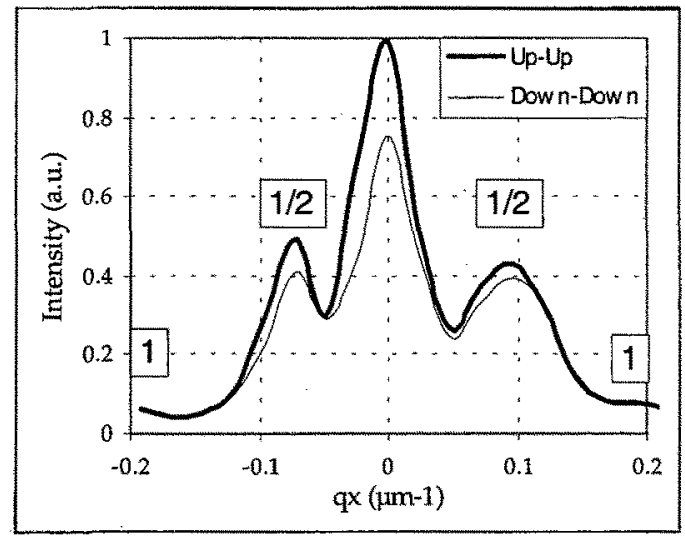

Figure 23 : rocking curve autour de $0.5^{\circ}$ sur un réseau de lignes de périodicité 5 um (lignes de largeur 1 um), sous un champ magnétique de $40 \mathrm{G}$. L'échantillon était incliné de $80^{\circ}$ par rapport au plan d'incidence lors de la mesture.

L'orientation de l'échantillon (inclinaison $\alpha$ de $80^{\circ}$ ) est telle que la période structurale vue par les neutrons est de $29 \mu \mathrm{m}$. Or les premiers pics satellites sont à une position située autour de $0.1 \mu^{-1}$, ce qui correspond en fait une période de $63 \mu \mathrm{m}$, double de la période structurale. Les pics satellites principaux sont donc des pics de superstructure d'ordre $1 / 2$, correspondant à un ordre antiferromagnétique entre les lignes du réseau.

Nous en déduisons que le retournement de l'aimantation dans ce réseau de lignes de $1 \mu \mathrm{m}$ a lieu par renversement de l'aimantation de certaines lignes qui se couplent antiferromagnétiquement aux lignes voisines. Au champ coercitif, le réseau se trouve dans un état où les lignes sont couplées antiferromagnétiquement entre elles. 


\subsection{Effet de l'énergie Zeeman dans les mesures de réflectivité de neutrons polarisés}

Lorsque l'on étudie des systèmes magnétiques, on est généralement amené à appliquer des champs magnétiques sur l'échantillon. Nous allons discuter l'effet de l'énergie Zeeman sur les mesures de réflectivité en neutrons polarisés et l'apparition de signaux hors des directions spéculaires. Cela nous permettra par ailleurs d'illustrer les techniques de mesure décrites précédemment. Les énergies mises en jeu par effet Zeeman sont très faibles (qqes $0.1 \mu \mathrm{eV}$ ), mais on va montrer qu'elles peuvent induire des modifications importantes sur les mesures de réflectivité et doit être prise en compte dans le cas de champs magnétiques appliqués forts (qqes $\mathrm{kG}$ ).

\subsubsection{Séparation spatiale des faisceaux de spin-flip et de non spin-flip}

Un des effets induit par l'énergie Zeeman est la séparation spatiale des faisceaux de neutrons de non spin-flip et ceux subissant un spin-flip (Felcher [9-10]). Nous allons l'illustrer ici par quelques exemples.

L'énergie d'un neutron dans un champ magnétique $\mathbf{H}$ est donnée par:

$$
E=\frac{\hbar^{2} k^{2}}{2 m} \pm g_{n} \mu_{n} H
$$

Le signe correspond à la polarisation du neutron par rapport au champ magnétique appliqué. Si l'énergie totale est conservée, un neutron polarisé $(+)$ ralentit en entrant dans un champ magnétique fort ; un neutron polarisé (-) accélère en entrant dans ce champ.

Dans une expérience de réflectivité, si l'aimantation du système étudié n'est pas parallèle à l'axe de quantification des neutrons (correspondant au champ extérieur appliqué), une fraction plus ou moins importante des neutrons réfléchis subit un « spin-flip » (cf. Cours précédent).

Considérons un échantillon placé dans un champ magnétique $\mathbf{H}$. Si lors de sa réflexion sur l'échantillon un neutron subit un spin-flip, la conservation de l'énergie dans un cas élastique permet d'écrire :

$$
\frac{\hbar^{2} k_{i}^{2}}{2 m}+g_{n} \mu_{n} H=\frac{\hbar^{2} k_{r}^{2}}{2 m}-g_{n} \mu_{n} H
$$

où $k_{i}$ et $k_{r}$ sont les vecteurs d'onde incident et réfléchi du neutron considéré. Le neutron subit un changement d'énergie $2 \mu_{n} H$ qui modifie son vecteur d'onde. Comme la composante planaire du vecteur d'onde est conservée lors de la réflexion (cf. Démonstration cours précédent), toute l'énergie est transférée sur la composante perpendiculaire $\mathrm{k}_{\mathrm{z}}$.

$$
k_{r}^{2}=k_{i}^{2} \pm \frac{4 m g_{n} \mu_{n} H}{\hbar^{2}}
$$

On peut calculer la relation entre l'angle d'incidence et l'angle de réflexion induit par ce changement de $\mathrm{k}_{\mathrm{z}}$ :

$$
\theta_{r}^{2}=\theta_{i}^{2} \pm \frac{4 m g_{n} \mu_{n}}{h^{2}} H \lambda^{2}
$$




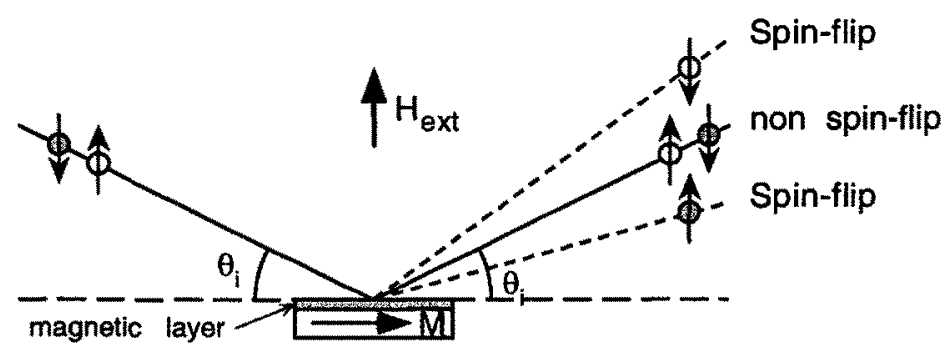

Figure 24 : sêparation spatiale des faisceaux de non spin-flip et de spin-flip induite par le changement d'énergie Zeeman lors d'un spin-flip.

Pour des champs de l'ordre de $0.5 \mathrm{~T}$, les effets sont très faibles pour $\lambda=0.4 \mathrm{~nm}$ (voir Tableau 1 ) mais les effets augmentent très vite avec la longueur d'onde (variation en $\lambda^{2}$ ). La déflexion des pics de spin flip atteint $0.1^{\circ}$ pour $\lambda=2 \mathrm{~nm}$.

Tableau 1 : angle de réflexion des faisceaux de spin-flip pour un angle d'incidence de $0.5^{\circ}$ sous un champ de $0.5 T$.

\begin{tabular}{cc}
\hline$\lambda_{\mathrm{i}}(\mathrm{nm})$ & $\theta_{\mathrm{r}}$ (degrés) \\
\hline 0.4 & 0.504 \\
1 & 0.524 \\
1.5 & 0.552 \\
2 & 0.589 \\
\hline
\end{tabular}

\subsubsection{Observation expérimentale (Felcher [9])}

Considérons une couche mince de cobalt. Il est possible d'appliquer des champs magnétiques de plusieurs $\mathrm{kG}$ perpendiculairement à la couche sans que l'aimantation de la couche mince ne sorte significativement hors du plan de la couche. En une couche mince de cobalt, le champ démagnétisant perpendiculairement à la couche est égal à $\mathrm{M}_{s}=1.7 \mathrm{~T}$ environ.

La figure suivante montre une mesure de la réflectivité sur cette couche de cobalt en fonction de l'état de spin incident. Cette mesure a été effectuée sur un spectromètre en temps de vol (voir Figure 13 pour le type de mesure). La mesure a été effectuée à un angle d'incidence constant $\theta_{i}=0.41^{\circ}$. Suivant une coupe à $\left(\theta_{i}+\theta_{i}=0.82^{\circ}\right)$, on observe le signal de réflectivité $(t+)$ sur la couche. On peut voir les oscillations successives due aux franges de Kiessig et dues à l'épaisseur finie de la couche. Ce qu'on peut observer en plus est l'apparition d'un signal hors spéculaire. Ce signal suit la trajectoire décrite par le calcul précédent. Dans le cas de neutrons incidents polarisés $(-)$, cette ligne de diffusion hors spéculaire apparât à la gauche (angles plus petits) du signal spéculaire. 

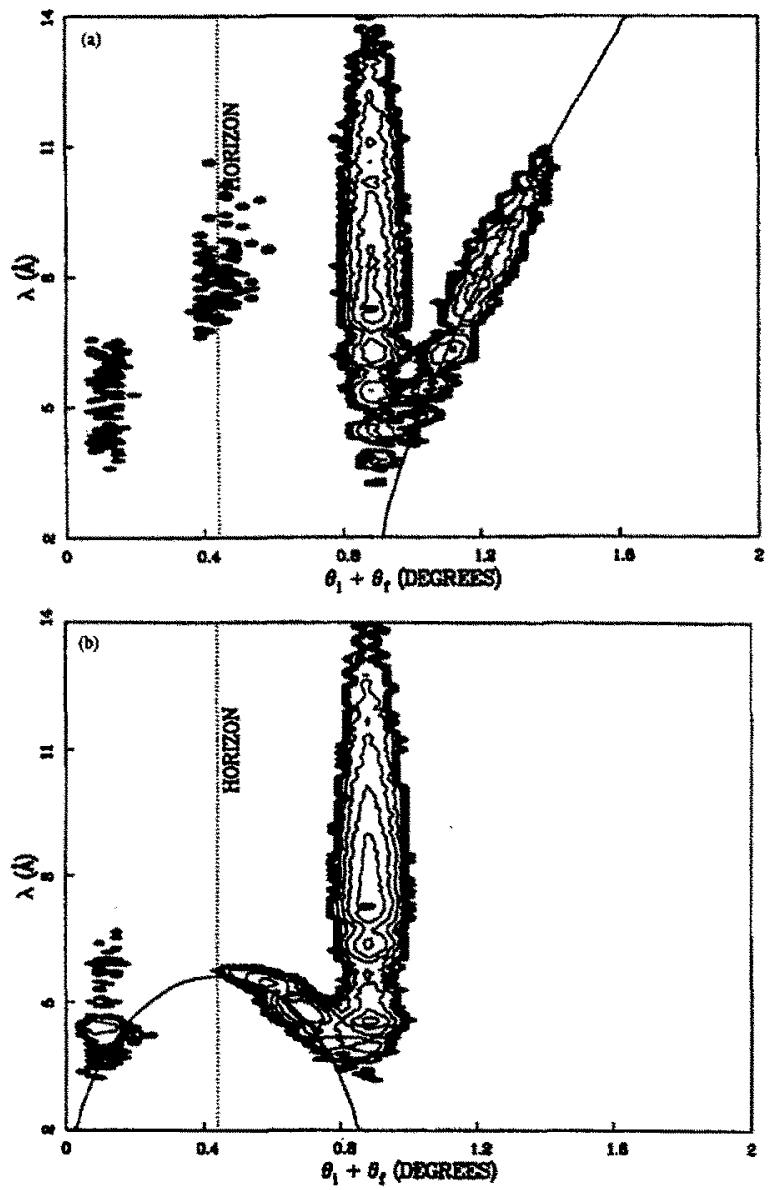

Figure 25 : intensités réfléchies sur une couche de cobalt dans un champ magnétique de $13 k G$. (a) neutron polarisés $(+) ;(b)$ neutrons polarisés $(-)$. Les intensités sont tracées en échelle log dans le plan $\left(\left(\theta_{i}+\theta_{r}\right) ; \lambda\right)$.

\subsubsection{Applications possibles}

Les énergies mises en jeu dans ces expériences sont extrêmement petits, de l'ordre de $10^{-7} \mathrm{eV}$. Cela montre la très grande sensibilité des mesures en incidence rasante. Cela pourrait suggérer une utilisation potentielle pour des mesures spectroscopiques d'excitation de surfaces mais de nombreux obstacles techniques existent. Aucune application n'a encore été réalisée. 


\subsection{Effet de la présence de domaines magnétiques sur le signal de spin-flip}

Considérons une couche mince magnétique démagnétisée présentant une structure en domaines magnétiques (voir Figure 26). Deux cas peuvent se présenter:

1. La zone d'illumination cohérente est plus petite que la taille caractéristique des domaines. Dans ce cas la réflexion sera donnée par la somme des réflexions spéculaires calculées pour les différentes orientations magnétiques des domaines. Comme il a été montré au cours précédent, lorsque qu'un neutron polarisé se réfléchi sur une couche mince dont l'aimantation $\mathbf{M}$ n'est pas parallèle à l'axe de quantification, il y a précession du spin dans la couche et donc la polarisation du neutron réfléchi est modifiée. Il y a en général apparition d'un signal de spin-flip important. Celui-ci est cependant parfaitement calculable.

2. La zone d'illumination cohérente est plus grande que la taille caractéristique des domaines. Dans ce cas, le calcul de la réflexion n'est pas simple. L'aimantation vue par le neutron n'est plus l'aimantation à saturation $\mathbf{M}_{\mathrm{s}}$ mais une valeur moyenne prise sur la zone illuminée de manière cohérente. Différentes "parties" de la fonction d'onde vont voir des champ locaux $\mathbf{M ( r )}$ différents. Cela conduit à des mécanismes de dépolarisation supplémentaires du neutron. Le calcul de la réflexion doit suivre les principes développés dans les techniques de dépolarisation (en transmission).

En pratique, les tailles de domaines sont généralement inférieures à quelques microns. Au contraire, la zone d'illumination cohérente est plutôt de l'ordre de quelques dizaines de $\mu \mathrm{m}$.
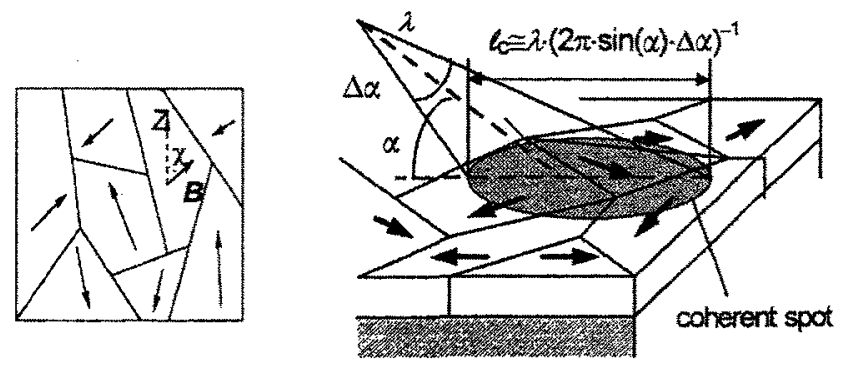

Figure 26: réflexion sur une couche présentant une structure en domaines magnétiques.(Pusenkov [11]).

\section{Nous allons présenter un exemple de mesure sur une multicouche $[\mathrm{Fe}(3 \mathrm{~nm}) / \mathrm{Cr}(\mathrm{Inm})]_{n}(\mathrm{Hahn}$,} [12]).

La Figure 27 présente les intensités diffusée sur la multicouche dans l'espace réciproque $\left(q_{z}, q_{x}\right)$ dans l'état vierge (a) et après un recuit à $350^{\circ} \mathrm{C}$ (b). Dans les deux cas, le champ appliqué est de 30G. On peut observer un signal hors spéculaire important autour du pic antiferromagnétique (AF) (qui est purement magnétique) de la structure multicouche alors qu'on observe pas de signal autour du pic de Bragg. Cela suggère que ce signal diffus est purement d'origine magnétique. A droite de la Figure 27, sont tracées deux coupes à $q_{z}$ constant correspondant à la position du pic AF.

La "largeur" du pic de diffraction AF dépend de la taille des domaines magnétiques. La largeur des pics est proportionnelle à un sinus cardinal au carré faisant intervenir la taille de ces domaines. Cette approximation est similaire à la technique utilisée pour évaluer la taille de petite particules par élargissement des raies en rayons $X$.

Dans le cas (a) état vierge, une taille de domaines $0.7 \mu \mathrm{m}$ peut être évaluée. Dans le cas (b) recuit, on observe une structure double: une structure large sur laquelle se superpose une pic relativement fin: on retrouve la taille de $0.7 \mu \mathrm{m}$ ainsi qu'une taille de $13 \mu \mathrm{m}$. Lorsque le champ magnétique 
augmente, les tailles de domaines sont inchangées mais l'intensité diffusée hors spéculaire diminue: les domaines de grande taille croissent au dépend des domaines de plus petite taille.
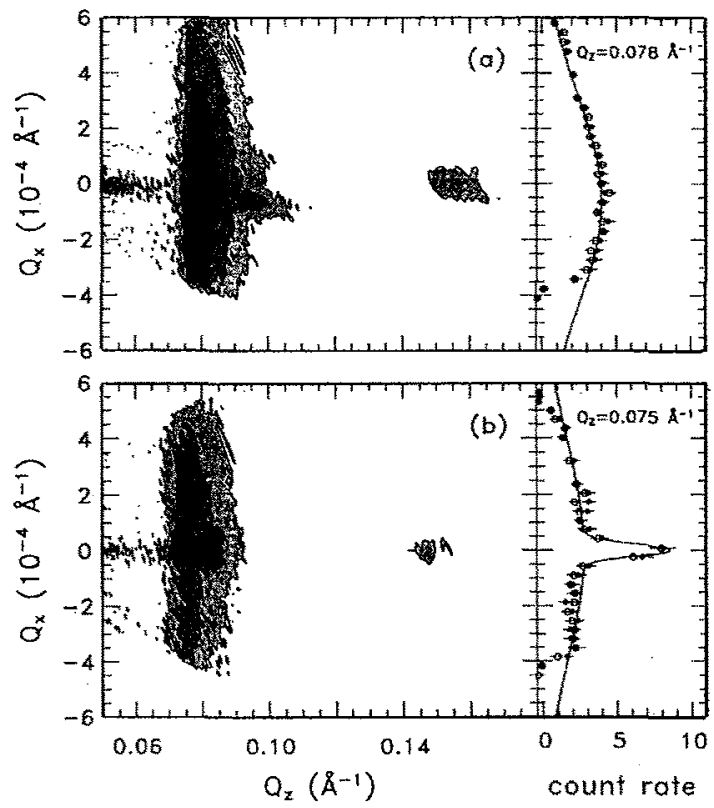

Figure 27: A gauche, intensité diffusée par les domaines d'une multicouches [Fe(3nm)/Cr(Inm)]; (a) comme déposée, (b) après recuit à $350^{\circ} \mathrm{C}$; A droite, coupe à $q_{z}$ constant sur le pic anti-ferromagnétique. (Hahn [12]).

\subsection{Diffraction de surface: ex. de l'étude d'une surface de fer (Gunther [13])}

Dans ce paragraphe, nous allons présenter un exemple de diffraction de surface sur une couche de fer épitaxiale. On verra que la diffraction magnétique sur cette structure en couche mince donne naissance à une structure en multiplet du pic de diffraction de surface. On montrera comment ce signal de diffraction donne des informations sur la structure magnétique du film et sur la rugosité magnétique de surface.

Considérons une couche magnétique d'aimantation $\mathbf{M}$. Il a été montré précédemment que le potentiel d'interaction du neutron avec cette couche magnétique est donné par: $V_{N} \pm \mu_{n} M$. Cette différence de potentiel d'interaction entre polarisation "up" et "down" donne naissance à un phénomène de biréfringence (voir Figure 28a): il y a séparation spatiale des directions de propagation $\mathrm{k}^{+}$et $\mathrm{k}^{-}$des neutrons polarisés "up" et "down". Comme corollaire, les angles d'incidence critiques $\alpha_{c \pm}$ sont différents en fonction de la polarisation. Un des points importants à noter est que le coefficient de transmission $t_{ \pm}$(pour les pol. "up" et "down") présente deux maxima distincts pour des angles d'incidence respectifs $\alpha_{c+}$ et $\alpha_{c-}$ (voir insert de la Figure 28a). Au niveau de l'interface Fer/substrat, un phénomène similaire de biréfringence apparait (pour $r_{ \pm}$).

En géométrie de diffraction de surface (voir Figure $28 \mathrm{~b}$ ), en raison de ces phénomènes de biréfringence, on s'attend donc à observer une séparation spatiale entre les différents états de spin $(++,-,+,-+)$. La structure du pic de Bragg doit présenter quatre maxima correspondant aux quatre combinaisons possibles $\alpha_{i, f}=\alpha_{c \pm}$. 
Pour les états de spin $(++)$ et $(-)$, les maxima apparaissent respectivement aux positions $\alpha_{i}=\alpha_{f}=\alpha_{c+}$ et $\alpha_{i}=\alpha_{f}=\alpha_{c-}$. Pour les états de spin-flip (+-) et (-+), les maxima apparaissent aux positions $\alpha_{i}=\alpha_{c+}, \alpha_{i}=\alpha_{c-}$ et $\alpha_{i}=\alpha_{c-}, \alpha_{i}=\alpha_{c+}$.

La Figure 28 (droite) présente une mesure expérimentale (Gunther [13], réflectomètre EVA, ILL) sur une couche de Fer(001) d'épaisseur 300nm déposée sur un substrat de $\mathrm{MgO}$ et terminée par une couche d'oxyde de Fer de $2.5 \mathrm{~nm}$. La figure représente les intensités diffusée en fonction de l'angle dincidence $\alpha_{i}$ et de réflexion $\alpha_{f}$ mesurées autour de la position du pic de diffraction (110). On observe bien la structure en multiplet, qui correspond à la séparation spatiale des quatre états de spin.

Il est possible de faire un calcul dans l'approximation de Born. Celui-ci permet de rendre compte de la position exacte des maxima et de la largeur des pics (voir Figure 28-droite-b). Cependant l'amplitude de diffraction du signal de spin-flip est beaucoup plus importante que ce qui est observé expérimentalement.

Les auteurs de cette mesure suggèrent que ce signal de spin-flip excédentaire peut s'expliquer par la rugosité magnétique au niveau de la couche d'oxyde. En effet, une rugosité de surface au niveau de la couche de fer donne naissance à des champs de fuite microscopiques $\delta B(\mathbf{r})$ au niveau de la surface (voir Figure 28-gauche-c).

Dans cette hypothèse, il est possible de mettre le facteur de structure sous la forme (pas de dém. disponible, voir Toperverg [14]):

$$
S(\boldsymbol{q})=b_{N}^{2}\left|a^{f}\right| \frac{\sigma_{B}}{d}\left[\left(1-g_{\perp}\right)\left(S_{++}+S_{--}\right)+g_{1}\left(s_{+-}+S_{+-}\right)\right]
$$

où $a^{f^{f}}=\left(q_{f_{+}}-q_{f_{-}}+q_{i_{+}}-q_{i_{-}}\right) d$ est un facteur de phase qui dépend de l'angle d'incidence, de l'angle de sortie et de l'épaisseur du film $d$. Les paramètres ajustables dans cette équation sont $\sigma_{B}$ la rugosité magnétique et $g_{\perp}$ la proportion de moments perpendiculaires à la surface. Un ajustement numérique montre que la rugosité magnétique s'étend sur une épaisseur deux fois supérieure à l'épaisseur de la couche d'oxyde superficielle. De plus on peut évaluer que $40 \%$ des moments de surface présentent des composantes perpendiculaires à la surface. 

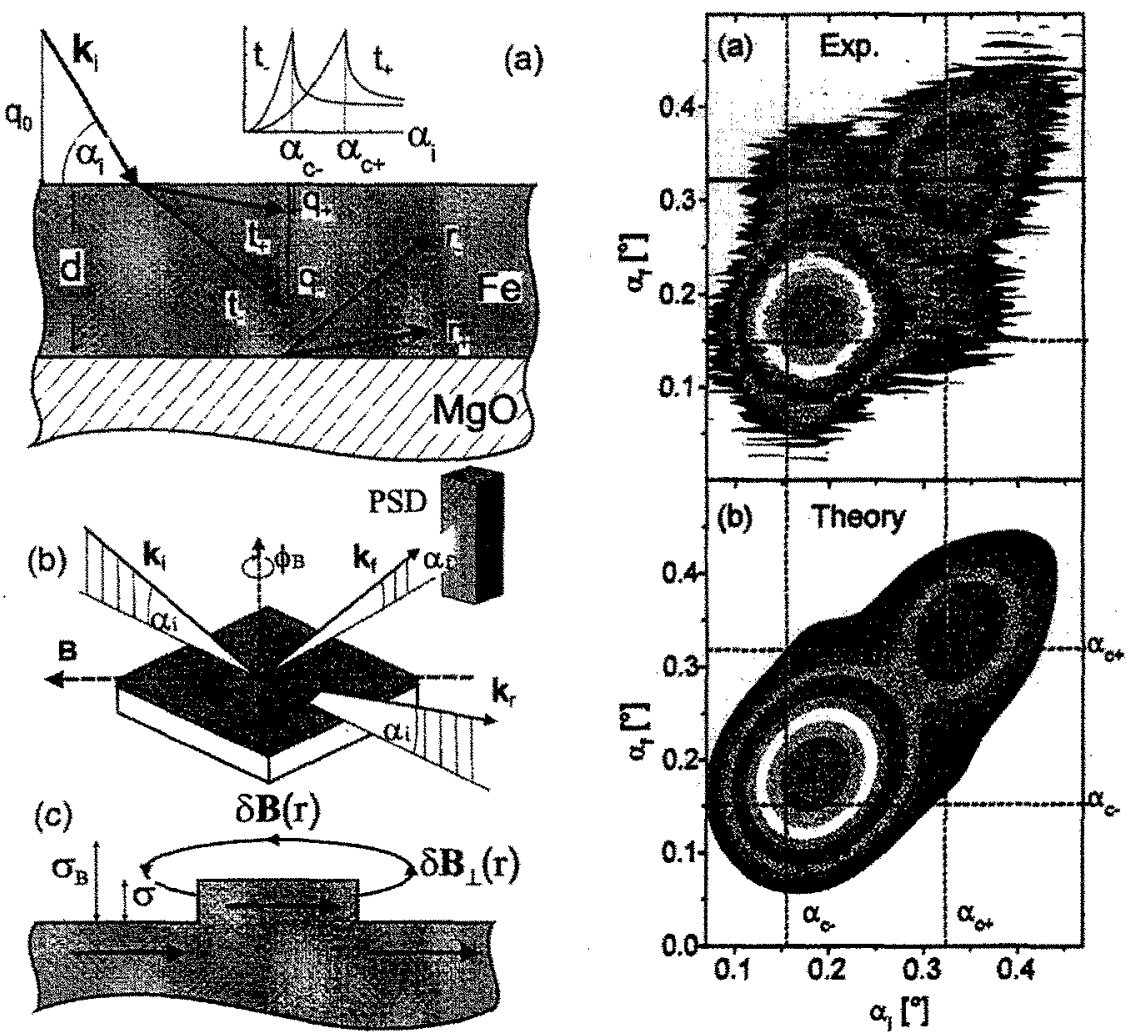

Figure 28: A gauche: (a) phénomène de réfraction dans une couche magnétique; (b) gêométrie de diffraction de surface; (c) structure magnétique de la couche de fer. A droite: signal hors spéculaire mesuré é calculé. (Günther, [13]).

\subsection{Diffusion sur un réseau de domaines magnétiques [15]}

Il a êté montré récemment par C. Fermon et A. Marty qu'il est possible d'envisager des mesures non spéculaires magnétiques sur des structures magnétiques «autoorganisées ». Les couches magnétiques de $\mathrm{Fe}_{50} \mathrm{Pd}_{50}$ sont des structures à aimantation perpendiculaire. Les domaines magnétiques dans ces systèmes peuvent s'organiser en lignes "magnétiques" de $50 \mathrm{~nm}$ de largeur environ. Un faible champ magnétique permet d'orienter ces domaines dans une direction privilégiée. La Figure 30 montre une image MFM de ces domaines orientés qui créent un réseau de lignes magnétiques dans une couche mince continue. Un réseau périodique magnétique de périodicité $100 \mathrm{~nm}$ est ainsi créé sans avoir recours aux techniques de lithographie.

Il est possible de réaliser des mesures de diffusion aux petits angles sur ces structures en lignes magnétiques. Une telle mesure a été réalisée sur le spectromètre de diffusion aux petits angles PAPOL du LLB par C. Fermon et A. Marty (voir géométrie sur la Figure 29). Dans l'expérience, les lignes sont alignées le long du plan d'incidence. La géométrie de diffraction est une géométrie de diffraction de surface. Le vecteur de diffusion possède deux composante : une composante « spéculaire » $q_{z}$ dans le plan d'incidence et une composante « hors spéculaire » $q_{y}$ perpendiculaire au plan d'incidence. L'aimantation des lignes est perpendiculaire à la composante $q_{y}$, ce qui permet de mesurer l'aimantation perpendiculaire dans ces couches de $\mathrm{Fe}_{50} \mathrm{Pd}_{50}$. 
Un exemple de diffraction est présenté sur la Figure 32. On peut observer un spot spéculaire principal et deux spots hors spéculaires d'intensité plus faible $\left(10^{-3}\right)$. Les positions de ces pics reflètent la périodicité des domaines magnétiques. Cependant, le maximum d'intensité de ces pics est obtenu dans la direction correspondant à l'angle critique de la couche quel que soit l'angle d'incidence. Le maximum d'intensité des pics hors spéculaires est obtenu lorsque l'angle d'incidence est égal à $\theta_{c}$. Ces pics ont un comportement similaires à des pics de Yoneda (Yoneda, [16]; Guentert, [17] ; Nigam, [18]).

Afin de décrire ces observations, on peut dans une première approche, utiliser l'approximation DWBA présentée en IV.A. Le système de départ «non perturbé » est la couche plane de $\mathrm{Fe}_{50} \mathrm{Pd}_{50}$; la perturbation est la structure magnétique créée par les domaines magnétiques. Dans ce cas, la section de diffusion est donnée par (Sinha [4]) :

$$
\left(\frac{d \sigma}{d \Omega}\right)_{\text {diff }}=\left(L_{x} L_{y}\right) \frac{\left|k_{0}^{2}\left(1-n^{2}\right)\right|^{2}}{16 \pi^{2}}\left|T\left(\mathbf{k}_{1}\right)\right|^{2}\left|T\left(\mathbf{k}_{2}\right)\right|^{2} S\left(\mathbf{q}_{t}\right)
$$

$\operatorname{avec} S\left(\mathbf{q}_{t}\right)=\frac{\exp \left(-\left[\left(q_{z}^{t}\right)^{2}+\left(q_{z}^{z^{*}}\right)^{2}\right] \sigma^{2} / 2\right)}{\left|q_{z}^{t}\right|^{2}} \iint_{S} d X d Y\left(\exp \left(\left|q_{z}^{t}\right|^{2} C(X, Y)\right)-1\right) \exp \left(i\left(q_{x} X+q_{y} Y\right)\right)$

où $C(X, Y)$ est la fonction de corrélation de la rugosité magnétique, $\mathbf{q}$ est le vecteur de diffusion $\mathbf{k}_{2-}$ $\mathbf{k}_{1}$ et $\mathbf{q}_{\mathrm{t}}$ est le vecteur de diffusion dans la couche. Des maxima dans la diffusion hors spéculaire sont obtenus lorsque les vecteurs $\mathbf{k}_{1}$ or $\mathbf{k}_{2}$ font un angle proche de $\theta_{c}$ car pour ces positions, les coefficients de Fresnel $\mathbf{T}$ sont maximaux.

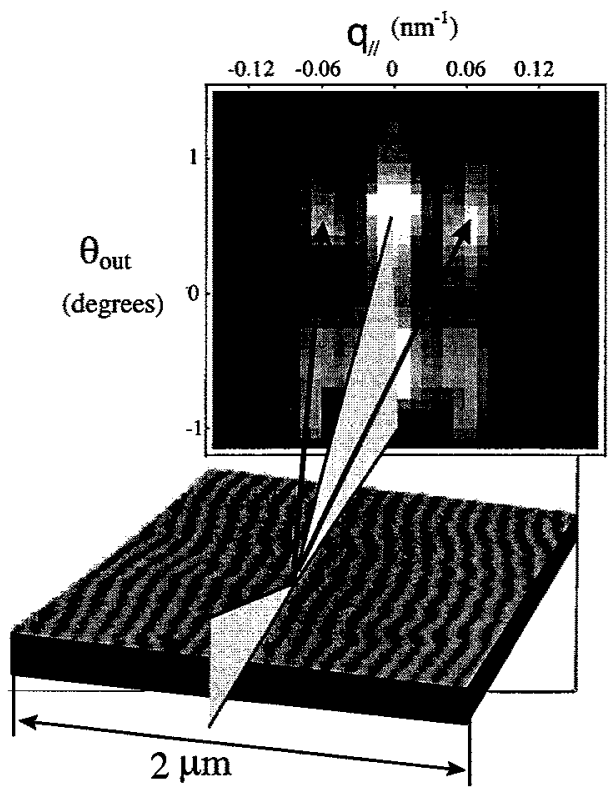

Figure 29: image MFM d'une couche mince magnétique FelPd présentant des domaines magnétiques orientés. Audessus, plan $2 D$ des intensités diffractées par le réseau de lignes (mesure réalisée sur le spectromètre PAPOL du $L L B$ ). (C. Fermon CEA/Saclay et A. Marty CEA/Grenoble). 
Dans le cas de lignes magnétiques, on définit la fonction de corrélation des lignes magnétiques par :

$$
C(X, Y)=\frac{1}{S_{0}} \iint_{s 0} M(x, y) M(x+X, y+Y) d x d y
$$

La fonction de corrélation est une fonction en dents de scie si on suppose des interfaces raides entre les domaines. La Figure 30 montre une image MFM et la fonction de corrélation correspondante. Comparé à l'image dans l'espace réel la fonction de corrélation présente des différences. La périodicité suivant (Oy) est la même que la périodicité des lignes mais les détails sont plus réguliers et les lignes sont plus droites.
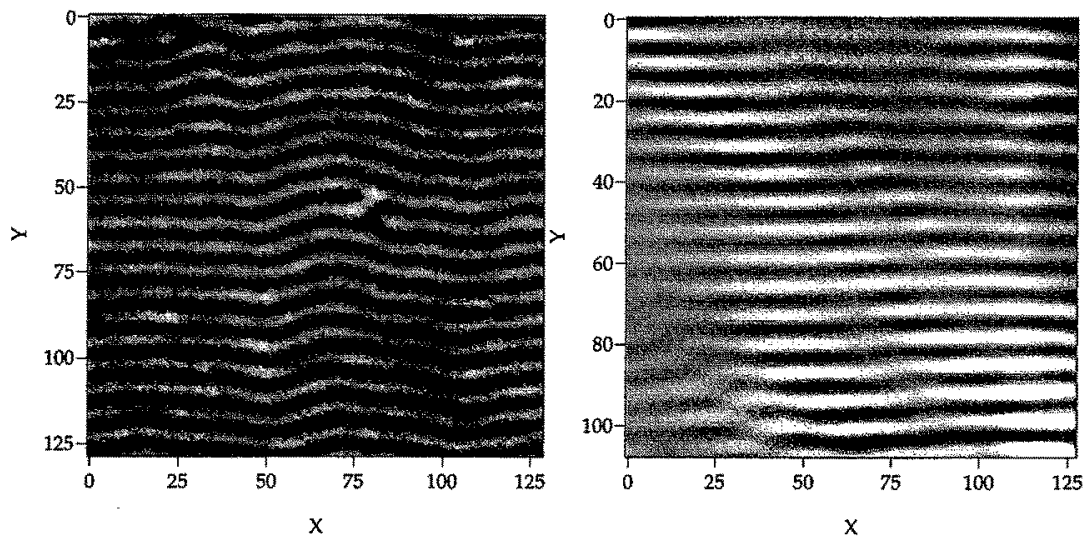

Figure 30 : image MFM à gauche (taille $2 \times 2 \mu \mathrm{m}$ ), fonction de « corrélation magnétique » correspondante à droite.

Si $q_{z}^{t}$ est petit, $S\left(\mathbf{q}_{z}\right)$ se réduit à la transformée de Fourier de la fonction de corrélation magnétique :

$$
S\left(q_{i}\right)=\iint_{S} d X d Y C(X, Y) \exp \left(i\left(q_{x} X+q_{y} Y\right)\right)
$$

Le signal hors-spéculaire mesure la transformée de Fourier de la fonction de corrélation magnétique. La Figure 31 montre la transformée de Fourier de la fonction de corrélation expérimentale ainsi que les deux coupes suivant les lignes $q_{x}=0$ et $q_{y}=0$. On peut remarquer que les deux pics de diffraction correspondent à la périodicité des lignes $(100 \mathrm{~nm})$ aux positions $\left(q_{x}=0\right.$, $q_{y}=0.62 \mathrm{~nm}^{-1}$ ). Cependant la forme de ces pics est légèrement asymétrique car les lignes magnétiques ne sont pas parfaitement alignées suivant $(\mathrm{Ox})$. Il y a par ailleurs un signal hors spéculaire assez important suivant $q_{x}=0$ ainsi que suivant $q_{y}=0$. Les pics apparaissant sur la figure n'ont rien à voir avec les pics de Yoneda, ils reflètent seulement la structure de la fonction de corrélation. 

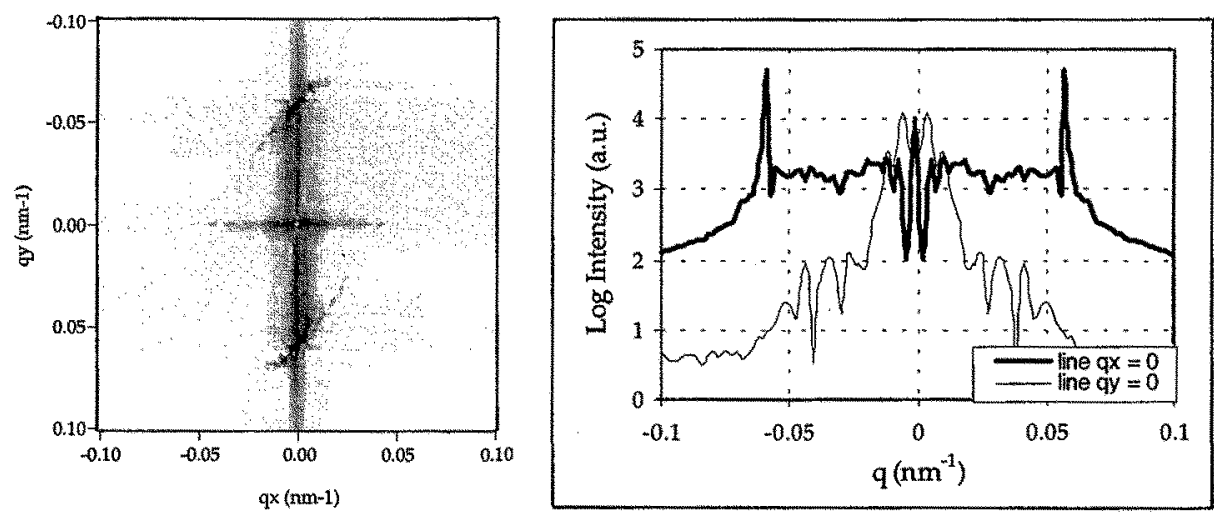

Figure 31 : à gauche, transformée de Fourier de la fonction de corrélation magnétique et à droite, deux coupes suivant les lignes $q_{x}=0$ et $q_{y}=0$

La Figure 32 montre le signal hors spéculaire calculé pour deux angles d'incidence différents, l'angle critique $\theta_{c}=0.5^{\circ}$ et $\theta=0.7^{\circ}$. Les positions des pics restent inchangés quel que soit l'angle d'incidence. Le maximum d'intensité est obtenu lorsque l'angle d'incidence est égal à l'angle critique $\theta_{c}$.

L'approche DWBA n'est cependant pas bien appropriée pour notre problème car la rugosité s'étend sur toute l'épaisseur de la couche magnétique de telle sorte que la couche plane n'est pas un bon état de base et est très loin des vrais états propres du système.

On insiste sur le fait que dans une expérience de diffraction de surface il est possible d'être sensible aux moments perpendiculaires à la surface.

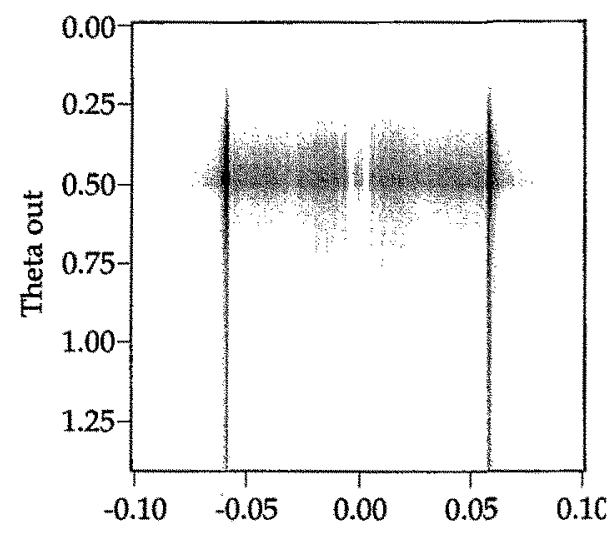

qy $(\mathrm{nm}-1)$

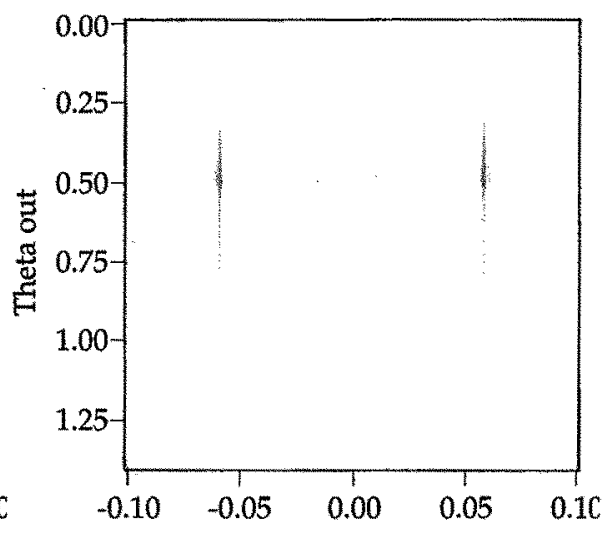

qy $(\mathrm{nm}-1)$

Figure 32 : signal hors spéculaire calculë pour deux angles d'incidences différents $(a) \theta=\theta_{c}=0.5^{\circ}$ et $(b) \theta=0.7^{\circ}$. La position des maxima ne bouge pas mais leur intensité décrô̂t lorsque l'angle d'incidence s'éloigne de de l'angle critique $\theta_{c}$ 


\subsection{Mesures SANS sur des couches minces}

Il existe quelques études de mesures SANS magnétique sur des films minces mais la limitation essentielle est l'interaction limitée avec le système. Cela impose de travailler de travailler sur une superposition de systèmes ou bien en incidence rasante. Un des problèmes est alors l'interaction avec le substrat qui peut générer un signal petits angles non négligeable.

Travaux récents: (Rekveldt, [19]), (Suzuki, [20]).

\subsection{Mesures de dépolarisation sur des films minces}

La technique de dépolarisation 3D a été appliquée à des films minces depuis de nombreuses années. Cette technique peut donner des informations sur la structure magnétique interne de films minces. En général, le mesure est faite en transmission ce qui limite sa sensibilité à des études de multicouches (Kraan [21-22]) ou d'empilement de nombreuses couches minces (Por [23]).

Pour le principe théorique des mesures de dépolarisation $3 \mathrm{D}$, nous renvoyons aux références suivantes :

(Rosman [24-25]) ou (Rekveldt [26]).

\section{CONCLUSION}

Toutes ces études de diffusion magnétique hors spéculaire sont encore très récentes et la thématique est en évolution constante. Les mesures de diffusion hors spéculaire magnétiques en sont encore à leur balbutiements. Cependant des progrès rapides peuvent être espérés lorsque des intensités plus importantes seront disponibles. Il sera alors possible d'effectuer des mesures précises sur des systèmes et dans des conditions variées afin de pouvoir comprendre tous les effets entrant en jeu dans ce type de diffraction (dont seulement une partie a été présentée ici).

\section{REFERENCES}

1. F. de Bergevin, X-ray and neutron reflectivity : principles and applications, Chap. 1 (ed. J.

Daillant and A. Gibaud, Springer, 1999).

2. H. Dosch et al. Rev. Sci Intr. 63, 5533 (1992); H. Dosch et al. Physica B 192, 163 (1993); H.

Dosch et al., Neutron News 6, 19 (1995).

3. H. Dosch, Critical phenomena at Surfaces and Interfaces; evanescent X-ray and neutron scattering (Springer Tracts in Modern Physics, vol 126, Berlin, 1992);

4. S.K. Sinha et al., Phys. Rev. B 38, 2297 (1988).

5. L. Mandel et al., Optical coherence and quantum optics (Cambridge University Press, 1995).

6. A. Messiah, Mécanique Quantique (Dunod, Paris, 1995).

7. J. Daillant, X-ray and neutron reflectivity : principles and applications, Chap. 4 (ed. J. Daillant and A. Gibaud, Springer, 1999).

8. J. Stettner, Dissertation : charakterisierung von rauchen MBE-CoSi2/Si Schichtsystemen (Kiel, 1995).

9. G.P. Felcher, S. Adenwalla, V.O. De Haan and A.A. Van Well, Nature 377, 409-410 (1995).

10. G.P. Felcher, S. Adenwalla, V.O. de Haan and A.A. Van Well, Physica B 221, 494-499 (1996).

11. V.M. Pusenkov, J.M.M.M. 175, 237 (1997). 
12. W. Hahn et al, J. Appl. Phys. 75, 3564 (1994).

13. R. Gunther et al., Phys. Rev. Lett. 81, 116 (1998).

14. B.P. Toperverg, Physica B 267-268, 198 (1999).

15. C. Fermon et al, Physica B 267-268, 162 (1999).

16. Y. Yoneda, Phys. Rev. B 131, 2010-2013 (1963).

17. O.J. Guentert, J. Appl. Phys. 30, 1361 (1965).

18. A.N. Nigam, Phys. Rev. A 4, 1189 (1965).

19. M. Th. Rekveldt et al. J.M.M.M. 78, 110 (1989).

20. J. Suzuki et al. J.M.M.M 184, 116(1998).

21. W.H. Kraan et al. J.M.M.M. 120, 372 (1993).

22. W.H. Kraan et al. Physica B 267-268, 75 (1999).

23. P.T. Por, J.M.M.M. 161, 357 (1996).

24. R. Rosman et al., Z. Phys B 79, 61 (1990).

25. R. Rosman et al. Phys. Rev. B 43, 8437 (1991).

26. M. Th. Rekveldt, Physica B 267-268, 60 (1999). 